Review

\title{
Actual and Perceived Motor Competence in Relation to Body Mass Index in Primary School-Aged Children: A Systematic Review
}

\author{
Athos Trecroci ${ }^{1, *(\mathbb{D})}$, Pietro Luigi Invernizzi ${ }^{1}\left(\mathbb{D}\right.$, Domenico Monacis $^{2}(\mathbb{D})$ and Dario Colella ${ }^{2} \mathbb{C}$ \\ 1 Department of Biomedical Sciences for Health, University of Milan, 20129 Milan, Italy; \\ pietro.invernizzi1@unimi.it \\ 2 Department of Humanities, Cultural Heritage, Education Sciences, University of Foggia, 71100 Foggia, Italy; \\ domenico.monacis@unifg.it (D.M.); dario.colella@unifg.it (D.C.) \\ * Correspondence: athos.trecroci@unimi.it
}

check for updates

Citation: Trecroci, A.; Invernizzi, P.L.; Monacis, D.; Colella, D. Actual and Perceived Motor Competence in Relation to Body Mass Index in Primary School-Aged Children: A Systematic Review. Sustainability 2021, 13, 9994. https://doi.org/ $10.3390 /$ su13179994

Academic Editor: Haywantee Ramkissoon

Received: 9 July 2021

Accepted: 3 September 2021

Published: 6 September 2021

Publisher's Note: MDPI stays neutral with regard to jurisdictional claims in published maps and institutional affiliations.

Copyright: (c) 2021 by the authors. Licensee MDPI, Basel, Switzerland. This article is an open access article distributed under the terms and conditions of the Creative Commons Attribution (CC BY) license (https:// creativecommons.org/licenses/by/ $4.0 /)$.

\begin{abstract}
Unhealthy-weight status may represent a precursor of poor actual (AMC) (i.e., processand product-oriented) and perceived (PMC) motor competence. AMC and PMC represent key elements favoring long-term commitment in sports and day-to-day life physical activity. In fact, the development of AMC and PMC during the primary school years could help to counteract weight-related negative effects (i.e., overweight or obesity) that are responsible for sedentary or unhealthy behavior across the life span. Therefore, this review aimed to provide a synopsis of the current research investigating the relation of AMC and PMC with weight status and in the context of potential gender differences. Systematic research in five electronic databases (PubMed, Web of Science, EMBASE, CINAHL, Scopus) was conducted from April 2021 to May 2021 in compliance with PRISMA guidelines. Studies were included if they involved obese or overweight youth (6-10 years) with no physical or cognitive impairment and used a longitudinal or a cross-sectional assessment of motor competence and perceived motor competence outcomes. After the selection process and after duplicates were removed, the final sample included 27 studies. Most of the studies reported that AMC and PMC are negatively associated with weight status, with male and females differing from each other in overall and subtest (locomotor, object control, and stability skills) AMC scores. However, according to a risk of bias assessment, the level of evidence linked to the association of AMC (process and product) and PMC with weight status (BMI) remained uncertain and lacking, respectively. Further high-quality studies are warranted to improve the understanding of AMC and PMC in relation to weight status, which appears to be differently expressed by gender in primary school years. Regardless, based on the current quantitative data, the emerging inverse association between AMC/PMC and weight status implies that it would be best to limit sedentary behavior by including daily lessons in physical education to limit unfavorable conditions (i.e., obesity and overweight) during the school years.
\end{abstract}

Keywords: overweight; pediatric obesity; childhood; motor skills

\section{Introduction}

Psychomotor development is critical for a child's motor and cognitive domains along with the youth continuum (e.g., from childhood and adolescence) [1]. The motor domain refers to children's ability to control the body for movement, while cognitive domain refers to specific mental abilities (e.g., attention and memory, reasoning, and perception) aimed at gathering and processing information [2]. Movement can occur with gross (e.g., walking) or fine (e.g., holding an object) gestures, depending on the use of large or small muscle groups [2]. For both gross- and fine-related skills, however, movement performance is linked to children's level of actual motor competence (AMC), which plays a key role in 
turning the health-related physical activity and sport participation in children's day-today lives into a long-term commitment [3,4]. A positive association between AMC and physical activity has been reported across childhood [3]. This association has also appeared for the cognitive domain; specifically, being able to perceive ones' own actual abilities contributes to prolonging the willingness to engage in sport or physical activity [5]. This may be mediated by self-regulatory mechanisms embracing intrinsic motivation and selfefficacy [6]. In this context, not only AMC but also perceived motor competence (PMC) may be of relevance for health-related lifestyle in children.

AMC encompasses several elements often ascribable to fundamental movement skills. Overall, these skills express the actual locomotor (e.g., running and hopping), object-control (e.g., catching and throwing), and stability (e.g., balancing and rotating) movement patterns [7] as precursors to more skill-demanding activities linked to sport disciplines [8]. Children presenting an insufficient acquisition of fundamental movement skills are less proficient (low AMC) and are likely to exhibit an altered perception (low PMC) of themselves [9]. As such, early, specific intervention strategies aimed at improving movement skills are desirable to level up a child's self-perception.

Together with environmental contexts (i.e., school, sports clubs, or place of living), low levels of AMC may lead to unhealthy behaviors (sedentary lifestyle) associated to weight and body mass index (BMI) increases. Specifically, for children, the daily school time spent on sedentary behavior is high ( $70 \%$ of school time), and, if not properly structured, school time might contribute to narrowing the opportunities for counteracting weightrelated negative effects [10]. Intuitively, overweight or obese children are likely to show lower AMC than normal-weight peers [11]. Overweight or obesity condition, compared to healthy-weight status, can impact the capability of a child to move their larger body mass against gravity [12], thereby also increasing clumsiness. Indeed, from a longitudinal perspective, the more a child's BMI increases, the less their AMC develops $[13,14]$. It has been consistently reported that children with unhealthy weight are expected to differ from those with healthy weight not only in gross motor skills but also in fine motor skills [15].

In this context, difficulties in maintaining a healthy-weight status may contribute to not overtaking a potential proficiency barrier. According to Seefeldt's original conception [16], a proficiency barrier refers to a critical level of motor competence, above or below which an individual will be more or less successful at learning advanced skills, respectively. This has implications on their health-related behavior across their lifespan in terms of both AMC and PMC. Notably, it has been observed that overall poor gross motor skills competence (i.e., bilateral coordination, upper limb coordination, balance, running speed, agility, and strength) was accompanied by poor physical abilities self-concept perception in overweight compared with nonoverweight middle-aged children [7]. Moreover, PMC also appears to impact children's future physical activities [17] as a consequence of a change in motivation in overweight and obese children [4,18].

Despite the body of literature indicating an inverse relationship between AMC/PMC and weight status (i.e., BMI values), evidence remains less informative in the youth continuum $[3,18]$. From a previous review article emerged that the extent of the inverse associations between AMC and BMI starts at early childhood (mainly preschool years) and turns more evident across middle childhood (mainly school years) [3]. However, results from another systematic review, 7 out of 12 studies found a weak association between AMC and BMI while 5 out of 12 studies did not found association in youngsters from early to late childhood (i.e., 3-13 years) [18]. Furthermore, similar inconclusive results are found for PMC [19-21] across childhood. LeGear et al. [19] and Robinson et al. [20] reported a positive association between PMC and AMC in early-aged children (4-5 years), while Valentini et al. [21] found a negative association in early- to late-aged children (3-10 years). These findings become more surprising if we consider the similar approaches adopted for assessing AMC and PMC, in terms of product- (e.g., Bruininks-Oseretsky Test of Motor Proficiency-2) and process-oriented (e.g., Test of Gross Motor Development-2) performance tests and perceived motor competence tests (e.g., Pictorial Scale of Perceived Competence). 
The role of gender differences in AMC and PMC in relation to weight status during school years should also be clarified. Such differences assume relevance in the context of a not-too-rare high degree of interindividual variation across childhood [22]. Apparently, girls are advantaged in their AMC if they encounter specific stimuli based on object control skills before the age of 10 [23]. On one hand, Barnett et al. [23] found no gender differences in locomotor skill proficiency. On the other hand, compared with boys, girls may mostly display lower AMC and PMC [24]. Regardless, girls are likely to receive little instruction in enhancing their fundamental movement skills as well as little opportunity to experience a variety of skills [23], which could affect their perception of how they move. Besides biological factors, the importance of environmental factors in driving potential gender differences poses the need to collectively clarify the extent of AMC and PMC levels within certain unfavorable conditions (e.g., unhealthy weight) during school years. Little and scattered information is known about gender-mediated differences in overweight and obese boys and girls during childhood. Because of the potential impact of the time during the school day on pursuing an active lifestyle as opposed to a sedentary behavior, it would be informative to clarify the association of AMC and PMC with weight status (i.e., BMI) in male (M) and female (F) children of primary school age. Moreover, this would also provide school personnel with additional and extended knowledge on health-related issues in the attempt to implement dedicated supporting programs of physical activity based on AMC and PMC enhancements.

Therefore, this review aimed to provide a synopsis of the current research investigating the relation of AMC and PMC with weight status, also in the context of potential gender differences, in an attempt to improve the understanding of the best intervention strategies to enhance AMC and PMC in M and F school-aged children.

\section{Materials and Methods}

\subsection{Eligibility Criteria and Study Selection}

The eligible studies were evaluated by title and abstract. Research was conducted separately by A.T. and P.I. according to the following criteria: (1) conducted in overweight and obese children from 6-12 years of age with no physical or cognitive impairment; (2) randomized controlled trials, intervention or longitudinal studies, and cross-sectional studies; (3) assessing of at least one of the following outcomes: product-oriented performance, process-oriented performance based on locomotor or object control skills or stability, and/or physical self-perception outcomes (components of academic, social, and emotional self-perception were not included); (4) limited to English language. There were excluded (1) studies that did not specify the sex of participants; (2) studies that evaluated only M or F; (3) studies including smaller (4-7 years) or larger (3-14 years) age ranges in which it was not possible to draw data from the age group of interest (from 6 to 12 years); (4) abstracts, dissertations, theses, and review articles.

\subsection{Information Sources and Search Strategy}

Comprehensive systematic research (from April 2021 to May 2021) was conducted for eligible studies published from 2011 in compliance with PRISMA guidelines [25,26] and drawing from the following databases: MEDLINE, Web of Science, Scopus, CINAHL, and EMBASE. Three groups of main concept terms ("Pediatric obesity AND Childhood AND psychomotor performance") were combined with each other and various other related keywords based on MEDLINE search terms, e.g., (1) "motor competen*", "motor coordination", "motor proficien*", "motor function", "motor skill"*", "sensimot", "movement

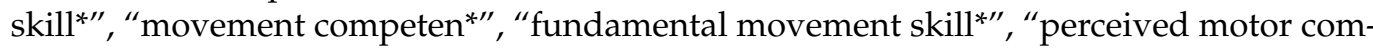
petence", "perceived sport* competence", "perceived competence", "perceived physical competence", "perception of competence", "perceived athletic competence"; (2) "body mass index", "obes", "overweigh"; (3) "child" ${ }^{* \prime, ~ " t e e n ", ~ a n d ~ " y o u t h " . ~}$ 


\subsection{Data Extraction and Risk of Bias Assessment}

Three researchers (A.T., P.I., and D.C.) extracted data from the selected papers. The extracted data included authors, year of publication, sample size, age (range), gender, weight status measures, AMC (product- and/or process-oriented assessment) or PMC measures, and study results. The risk of bias was assessed in compliance with the STROBE [27] and CONSORT [28] guidelines as previously adopted by Lubans et al. [29]. A six-point scale was used to provide a quality score, which referred to 0 (absent or inadequately described) or 1 (explicitly described and present) values assigned to each of the following questions: (i) did the study describe the participants' eligibility criteria?; (ii) were the participants randomly selected?; (iii) did the study report the sources and details of AMC or PMC assessment, and did the instruments have acceptable reliability for the specific age group?; (iv) did the study report the sources and details of assessment of potential benefits, and did all of the methods have acceptable reliability for the specific age group?; (v) did the study report a power calculation, and was the study adequately powered to detect hypothesized relationships?; and (vi) did the study report the numbers of individuals who completed each of the different measures and did participants complete at least $80 \%$ of AMC or PMC and benefit measures? Those studies scoring $\leq 2$ were considered to have high risk of bias. Those scoring 3-4 points were considered to have medium risk, while those scoring 5-6 points were considered to have low risk. In the present study, the term association was used to express a broad traversal concept, not only referring to the measure of the specific relationship between two variables (e.g., inverse relationship of AMC and BMI in individuals), but also to suggest the general idea that both variables covary (e.g., healthy-weight individuals present higher AMC than unhealthy-weight peers).

\section{Results}

The review process yielded 2629 records from six electronic databases (Figure 1). After a preliminary removal before screening (e.g., duplicate removal), a total of 27 articles met the inclusion criteria and were included in the final review process. The overall number of participants was $8548 \mathrm{~F}$ (ranging from 21 to 1826) and $8928 \mathrm{M}$ (ranging from 26 to 1912), aged from 6 to 12 years.

\subsection{Study Characteristics and Quantitative Data Interpretation}

Eleven [21,30-39] studies employed an AMC assessment based on process-oriented tests. Specifically, four studies used the Test of Gross Motor Development, second edition (TGMD-2), while only one study used the third edition (TGMD-3). Three studies used the "Move it Groove it" process-oriented checklists, while only one study used the Get-skilled Get-active process-oriented checklists. One study used the PLAYbasic and Passport for Life fundamental movement skill tools (see Table 1).

Regarding AMC assessment based on product-oriented tests, nine studies used the Körperkoordinationstest für Kinder (KTK). Four studies used the Bruininks-Oseretsky Test of Motor Proficiency Short Form (BOTMP-SF), while two studies used the second edition (BOTMP-2). Only one study used the Movement Assessment Battery for Children, second edition (MABC-2). Only one study used three product-oriented tests encompassing locomotor skill, speed, and agility (see Table 1). 
Table 1. Resume of the studies linked to the association between actual (AMC) and perceived (PMC) motor competence and weight status.

\begin{tabular}{|c|c|c|c|c|c|c|}
\hline \multirow{2}{*}{ Author } & \multirow{2}{*}{ Subjects } & \multicolumn{2}{|c|}{ AMC Measure } & \multirow{2}{*}{ PMC Measure } & \multirow{2}{*}{$\begin{array}{l}\text { Weight } \\
\text { Status } \\
\text { Measure }\end{array}$} & \multirow{2}{*}{ Results } \\
\hline & & Process-Oriented & Product-Oriented & & & \\
\hline Augustijn et al. [40] & $\begin{array}{c}21 \mathrm{~F} \\
33 \mathrm{M} \\
(7-11 \mathrm{yrs}) \\
\end{array}$ & $\mathrm{n} / \mathrm{a}$ & $\begin{array}{l}\text { MABC-2 }(3 \text { subscales, including manual } \\
\text { dexterity skills, such as posting coins, } \\
\text { threading beads, and drawing; ball skills, } \\
\text { such as catching with two hands and } \\
\text { throwing beanbag onto mat; and total } \\
\text { balance skills, such as one-leg balance, } \\
\text { walking heels raised, and jumping): total } \\
\text { and subscale scores }\end{array}$ & $\mathrm{n} / \mathrm{a}$ & $\begin{array}{l}\text { BMI, FM, } \\
\text { and WC }\end{array}$ & $\begin{array}{l}\text { (i) OB children's level of motor competence was lower than that of } \\
\text { HW children }(p<0.001) \text { in terms of manual dexterity }(p=0.014) \\
\text { and static and dynamic balance }(p<0.001) \text {; ii) no significant } \\
\text { difference was found for ball skills. }\end{array}$ \\
\hline Bryant et al. [30] & $\begin{array}{c}152 \mathrm{~F} \\
129 \mathrm{M} \\
(6-11 \mathrm{yrs})\end{array}$ & $\begin{array}{l}\text { POC applied to “Move it Groove it" } \\
\text { program (LOC skills: sprint, hop, vertical } \\
\text { jump, and gallop; OC skills: kick, catch, } \\
\text { and overarm throw): LOC and OC } \\
\text { subtest scores. }\end{array}$ & $\mathrm{n} / \mathrm{a}$ & $\mathrm{n} / \mathrm{a}$ & BMI & $\begin{array}{l}\text { (i) BMI affected only the sprint run }(p=0.002) \text {. (ii) } 8-9 \text { yrs: LOC } \\
\text { skills dropped in mastery level }(p<0.05) \text {. (iii) } 9-10 \text { yrs: } 2 / 3 \text { OC } \\
\text { skills (catch and throw) dropped in mastery level }(p<0.05) \text {; (iv) M } \\
\text { kicked and threw better than F children }(p<0.05) \text {, while F were } \\
\text { significantly better in balance than M children. }\end{array}$ \\
\hline Bryant et al. [31] & $\begin{array}{c}152 \mathrm{~F} \\
129 \mathrm{M} \\
(6-11 \mathrm{yrs})\end{array}$ & $\begin{array}{l}\text { POC applied to "Move it Groove it" } \\
\text { program (LOC skills: sprint, hop, vertical } \\
\text { jump, and gallop; OC skills: kick, catch, } \\
\text { and overarm throw): total FMS score and } \\
\text { LOC and OC subtest scores. }\end{array}$ & $\mathrm{n} / \mathrm{a}$ & $\mathrm{n} / \mathrm{a}$ & BMI, FM & $\begin{array}{l}\text { (i) FMS score was a good predictor of } \mathrm{BMI} \text { and } \mathrm{FM} \text {; ii) } \mathrm{M} \text { kicked } \\
\text { and threw better than } \mathrm{F} \text { children }(p<0.05) \text {, while } \mathrm{F} \text { were } \\
\text { significantly better in balance than M children. }\end{array}$ \\
\hline Cliff et al. [32] & $\begin{array}{l}73 \mathrm{~F}, 59 \mathrm{M} \\
(6-10 \mathrm{yrs}) \\
\end{array}$ & $\begin{array}{l}\text { TGMD-2 (LOC skills such as run, gallop, } \\
\text { hop, leap, jump, and slide, and OC skills } \\
\text { such as strike, dribble, catch, kick, throw, } \\
\text { and underhand roll): prevalence (\%) of } \\
\text { LOC and OC skill mastery and prevalence } \\
\text { of advanced LOC and OC skill proficiency. }\end{array}$ & $\mathrm{n} / \mathrm{a}$ & $\mathrm{n} / \mathrm{a}$ & $\begin{array}{l}\text { BMI, BMI, } \\
\text { and z-scores }\end{array}$ & $\begin{array}{l}\text { The prevalence of mastery was significantly higher in HW } \\
\text { compared with OW or OB children for both LOC and OC skills } \\
\text { between 6-10 yrs. Overall, this result applied for advanced } \\
\text { skill proficiency. }\end{array}$ \\
\hline Cheng et al. [41] & $\begin{array}{c}307 \mathrm{~F} \\
361 \mathrm{M} \\
(10 \mathrm{yrs})\end{array}$ & $\mathrm{n} / \mathrm{a}$ & $\begin{array}{l}\text { BOTMP-SF ( } 6 \text { subtests of gross motor skills, } \\
6 \text { subtests of fine motor skills, and } \\
2 \text { subtests of both gross and fine motor } \\
\text { skills): total score of motor proficiency and } \\
\text { gross and fine motor skill scores. }\end{array}$ & $\mathrm{n} / \mathrm{a}$ & $\begin{array}{c}\text { BMI, } \\
\text { z-scores }\end{array}$ & $\begin{array}{l}\text { (i) BMI z-scores and total scores were negatively correlated } \\
(p<0.05) ; \text { (ii) OB and OW differed from HW children in total and } \\
\text { gross motor proficiency; (iii) there were no group differences on } \\
\text { any of the fine motor subtests at age 10; (iv) M exhibited a higher } \\
\text { total score than F children. }\end{array}$ \\
\hline Chowdhury et al. [42] & $\begin{array}{c}398 \mathrm{~F} \\
418 \mathrm{M} \\
(5-12 \mathrm{yrs})\end{array}$ & $\mathrm{n} / \mathrm{a}$ & $\begin{array}{l}\text { BOTMP-SF ( } 6 \text { subtests of gross motor skills, } \\
6 \text { subtests of fine motor skills, and } 2 \\
\text { subtests of both gross and fine motor skills): } \\
\text { total motor proficiency standard score. }\end{array}$ & $\mathrm{n} / \mathrm{a}$ & $\begin{array}{c}\text { BMI, } \\
\text { z-scores }\end{array}$ & $\begin{array}{l}\text { (i) BMI was associated with motor competence of children } \\
\text { ( } p<0.01) \text {; (ii) HW children had higher motor competence scores } \\
\text { compared to UW (OB and OW) children in total BOT-2 score }\end{array}$ \\
\hline Comeau et al. [33] & $\begin{array}{c}67 \mathrm{~F} \\
78 \mathrm{M} \\
(9-12 \mathrm{yrs}) \\
\end{array}$ & $\begin{array}{l}\text { PLAYbasic (run there and back, hop, } \\
\text { overhead throw, kick ball, and balance } \\
\text { walk backwards). Passport for Life (lateral } \\
\text { bound, plank, run-stop-return, throw and } \\
\text { catch, kicks, and a four-station circuit): } \\
\text { PLAYbasic and Passport for Life scores. }\end{array}$ & $\mathrm{n} / \mathrm{a}$ & $\mathrm{n} / \mathrm{a}$ & $\begin{array}{l}\text { BMI, } \\
\text { z-scores, FM, } \\
\text { and WC }\end{array}$ & $\begin{array}{l}\text { (i) PLAYbasic score was inversely associated with WC }(p<0.05) \\
\text { and FM ( } p=0.001) \text { but not with BMI. LOC skills (run there and } \\
\text { back and hop) were inversely }(p=0.001) \text { related with BMI, while } \\
\text { OC (overhead throw, kick ball) and stability skills (balance walk } \\
\text { backwards) were not. (ii) Passport for Life score was inversely } \\
\text { associated with BMI }(p<0.05) \text { and FM }(p=0.001) \text {, while no } \\
\text { association was found with WC }(p>0.05) \text {. No significant } \\
\text { association was found between OC skills and BMI. }\end{array}$ \\
\hline Coppens et al. [43] & $\frac{265 \mathrm{~F}}{293 \mathrm{M}}$ & $\mathrm{n} / \mathrm{a}$ & $\begin{array}{l}\text { KTK (balancing backward, jumping } \\
\text { laterally, hopping one leg, and shifting } \\
\text { platforms): total MQ score }\end{array}$ & $\mathrm{n} / \mathrm{a}$ & BMI & $\begin{array}{l}\text { (i) BMI was negatively associated with the KTK MQ score at } \\
\text { baseline ( } 6 \text { yrs old) and also with its change across } 2 \text { yrs ( } 9 \text { yrs old); } \\
\text { (ii) F made less progress in the KTK MQ than M children across } \\
2 \text { yrs, but had no difference at baseline. }\end{array}$ \\
\hline
\end{tabular}


Table 1. Cont.

\begin{tabular}{|c|c|c|c|c|c|c|}
\hline \multirow{2}{*}{ Author } & \multirow{2}{*}{ Subjects } & \multicolumn{2}{|c|}{ AMC Measure } & \multirow{2}{*}{ PMC Measure } & \multirow{2}{*}{$\begin{array}{c}\text { Weight } \\
\text { Status } \\
\text { Measure }\end{array}$} & \multirow{2}{*}{ Results } \\
\hline & & Process-Oriented & Product-Oriented & & & \\
\hline \multirow{3}{*}{ De Meester et al. [34] } & $181 \mathrm{~F}$ & \multirow{3}{*}{$\begin{array}{l}\text { TGMD-2 (LOC skills such as run, gallop, } \\
\text { hop, leap, jump, and slide, and OC skills } \\
\text { such as strike, dribble, catch, kick, throw, } \\
\text { and underhand roll): TGMD-2 total score. }\end{array}$} & \multirow{3}{*}{$\mathrm{n} / \mathrm{a}$} & \multirow{3}{*}{$\begin{array}{l}\text { Self-Perception } \\
\text { Profile for Children } \\
\text { (sport/athletic } \\
\text { subscale): } \\
\text { PMC score }\end{array}$} & \multirow{3}{*}{ BMI } & \multirow{3}{*}{$\begin{array}{l}\text { (i) Total score was positively }(p<0.001) \text { correlated with PMC and } \\
\text { inversely }(p<0.05) \text { correlated with BMI; (ii) PMC was inversely } \\
(p<0.001) \text { correlated with BMI; (iii) total score did not differ } \\
\text { between M and F children, but M reported higher PMC scores than } \\
\text { F children }(p=0.04) \text {. }\end{array}$} \\
\hline & $180 \mathrm{M}$ & & & & & \\
\hline & $(6.92-11.83$ yrs $)$ & & & & & \\
\hline \multirow[b]{2}{*}{ Dos'Santos et al. [44] } & $232 \mathrm{~F}$ & \multirow[b]{2}{*}{$\mathrm{n} / \mathrm{a}$} & \multirow{2}{*}{$\begin{array}{l}\text { KTK (balancing backward, jumping } \\
\text { laterally, hopping one leg, and shifting } \\
\text { platforms): individual gross motor } \\
\text { test score }\end{array}$} & \multirow[b]{2}{*}{$\mathrm{n} / \mathrm{a}$} & \multirow[b]{2}{*}{$\begin{array}{l}\text { BMI, FM } \\
\text { and FFM }\end{array}$} & \multirow{2}{*}{$\begin{array}{l}\text { (i) There were significant inverse associations of BMI and FM with } \\
\text { balancing backward, jumping laterally, hopping one leg, and } \\
\text { shifting platforms, and between FFM and balancing backwards; } \\
\text { (ii) there were positive associations between FFM and jumping } \\
\text { laterally, hopping one leg, and shifting platforms }(p<0.05) \text {. }\end{array}$} \\
\hline & $251 \mathrm{M}$ & & & & & \\
\hline \multirow[t]{3}{*}{ D’Hondt et al. [45] } & $500 \mathrm{~F}$ & \multirow[t]{3}{*}{$\mathrm{n} / \mathrm{a}$} & \multirow{3}{*}{$\begin{array}{l}\text { KTK (balancing backward, jumping } \\
\text { laterally, hopping one leg, and shifting } \\
\text { platforms): total and individual MQ } \\
\text { test score }\end{array}$} & \multirow[t]{3}{*}{$\mathrm{n} / \mathrm{a}$} & \multirow[t]{3}{*}{ BMI } & \multirow{3}{*}{$\begin{array}{l}\text { (i) For 8-9 yrs and 10-12 yrs, HW children showed higher total } \\
\text { scores than OW and OB children }(p<0.05) ;(\text { ii) for } 8-9 \text { yrs and } \\
10-12 \text { yrs, HW children outperformed both OW and OB children } \\
(p<0.001) \text { in balance- and hop-based KTK tests }(p<0.001) ; \text { iii) only } \\
\text { for 10-12 yrs, HW children outperformed OW }(p=0.012) \text { and OB } \\
(0.007) \text { children in jumping laterally; iv) M children were better at } \\
\text { jumping and shifting platforms, while F children were better in } \\
\text { balancing backwards. }\end{array}$} \\
\hline & $454 \mathrm{M}$ & & & & & \\
\hline & $(\sim 6-12$ yrs $)$ & & & & & \\
\hline \multirow[b]{2}{*}{ D’Hondt et al. [46] } & $24 \mathrm{~F}$ & \multirow[b]{2}{*}{$\mathrm{n} / \mathrm{a}$} & \multirow{2}{*}{$\begin{array}{l}\text { KTK (balancing backward, jumping } \\
\text { laterally, hopping one leg, and shifting } \\
\text { platforms): total MQ and individual } \\
\text { test score }\end{array}$} & \multirow[b]{2}{*}{$\mathrm{n} / \mathrm{a}$} & \multirow{2}{*}{$\begin{array}{l}\text { BMI, BMI, } \\
\text { z-scores, and } \\
\text { FM }\end{array}$} & \multirow{2}{*}{$\begin{array}{l}\text { (i) At } \sim 8 \text { (baseline) and } \sim 10 \text { yrs ( } 2 \text { yrs follow-up), total score was } \\
\text { inversely related with BMI, BMI } \text {-scores, and FM; (ii) BMI at } \\
\text { baseline alone explained } 37.6 \% \text { of the variance in KTK gross motor } \\
\text { skills (LOC and stability skills); (iii) OB and OW children } \\
\text { presented lower total score }(p<0.05) \text { than HW children. }\end{array}$} \\
\hline & $26 \mathrm{M}$ & & & & & \\
\hline \multirow{3}{*}{ D’Hondt et al. [47] } & 24F $48 \mathrm{M}$ & \multirow{3}{*}{$\mathrm{n} / \mathrm{a}$} & \multirow{3}{*}{$\begin{array}{l}\text { KTK (balancing backward, jumping } \\
\text { laterally, hopping one leg, and shifting } \\
\text { platforms): total MQ and individual } \\
\text { test score }\end{array}$} & \multirow{3}{*}{$\mathrm{n} / \mathrm{a}$} & & (i) $\mathrm{OW}$ or $\mathrm{OB}$ children showed poorer $\mathrm{MQ}$ scores than $\mathrm{HW}$ \\
\hline & $24 \mathrm{~F}, 48 \mathrm{MI}$ & & & & $\begin{array}{l}\text { BMI, FM, } \\
\text { and WC }\end{array}$ & $\begin{array}{l}\text { children from baseline to } 4 \text { months after } \mathrm{OB} \text { treatment; (ii) } \mathrm{MQ} \text { and } \\
\text { individual (LOC and stability skills) scores were different in }\end{array}$ \\
\hline & $10-11$ yrs & & & & & $\begin{array}{l}\text { relation to BMI (HW versus OW/OB children). } \\
\text { rent in }\end{array}$ \\
\hline Duncan et al. [35] & $136 \mathrm{~F}$ & $\begin{array}{l}\text { POC applied to "Move it Groove it" } \\
\text { program (locomotor skills: sprint, hop, } \\
\text { vertical jump and gallop; object control } \\
\text { skills: kick, catch and overarm throw): total }\end{array}$ & 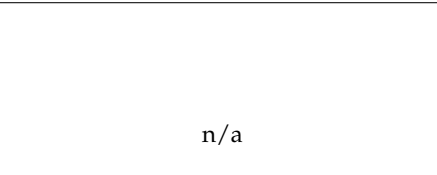 & $\mathrm{n} / \mathrm{a}$ & $\mathrm{BMI}$ and FM & $\begin{array}{l}\text { (i) For BMI, children classed as having low LOC skills had higher } \\
\text { BMI compared to those classed as having medium }(p=0.006) \text { or } \\
\text { high }(p=0.0001) \text { FMS proficiency; ii) for FM, children classed as } \\
\text { having low LOC skills had higher FM compared to those classed } \\
\text { as having medium }(p=0.021) \text { or high ( } p=0.0001) \text { FMS proficiency; } \\
\text { iii) no difference was found in BMI or FM as a function of FMS }\end{array}$ \\
\hline & $112 \mathrm{M}$ & $\begin{array}{l}\text { FMS score, and locomotor and object } \\
\text { control scores }\end{array}$ & & & & $\begin{array}{l}\text { proficiency for OC skills; iv) in F children, BMI was lower for } \\
\text { children with high and medium total FMS scores compared to }\end{array}$ \\
\hline & $(6-11 \mathrm{yrs})$ & & & & & those with low FMS proficiency $(p=0.015, p=0.027)$ \\
\hline & $29 \mathrm{~F}$ & $\begin{array}{l}\text { The Functional Movement Screen (seven } \\
\text { tests such as deep squat, hurdle step, }\end{array}$ & & & & (i) Total FMS score was negatively correlated with BMI ( $p<0.001)$; \\
\hline $\begin{array}{l}\text { Duncan and } \\
\text { Stanley [36] }\end{array}$ & $29 \mathrm{M}$ & in-line lunge, shoulder mobility, active & $\mathrm{n} / \mathrm{a}$ & $\mathrm{n} / \mathrm{a}$ & BMI & $\begin{array}{l}\text { (ii) HW children scored better than OW and OB peers }(p=0.0001) \text {; } \\
\text { (iii) no differences were observed in FMS scores between } \mathrm{M} \text { and }\end{array}$ \\
\hline & $(10-11 \mathrm{yrs})$ & $\begin{array}{l}\text { straight leg raise, trunk-stability push-up, } \\
\text { and rotary stability): total FMS score }\end{array}$ & & & & F children. \\
\hline
\end{tabular}


Table 1. Cont.

\begin{tabular}{|c|c|c|c|c|c|c|}
\hline \multirow{2}{*}{ Author } & \multirow{2}{*}{ Subjects } & \multicolumn{2}{|c|}{ AMC Measure } & \multirow{2}{*}{ PMC Measure } & \multirow{2}{*}{$\begin{array}{l}\text { Weight } \\
\text { Status } \\
\text { Measure }\end{array}$} & \multirow{2}{*}{ Results } \\
\hline & & Process-Oriented & Product-Oriented & & & \\
\hline Gentier et al. [15] & $\begin{array}{c}24 \mathrm{~F} \\
44 \mathrm{M} \\
(7-\sim 12 \mathrm{yrs}) \\
\end{array}$ & $\mathrm{n} / \mathrm{a}$ & $\begin{array}{l}\text { BOTMP-2 ( } 8 \text { subtests, including fine motor } \\
\text { precision and integration, manual dexterity, } \\
\text { upper-limb coordination, bilateral } \\
\text { coordination, balance, running speed and } \\
\text { agility, and strength): total BOTMP- } 2 \text { and } \\
\text { individual gross and fine scores }\end{array}$ & & BMI & $\begin{array}{l}\text { (i) HW children were superior to their OB peers in total BOTMP-2 } \\
\text { score }(p<0.001) ;(\text { ii) HW children scored higher for gross and fine } \\
\text { motor subtests compared than OB children; (iii) HW children did } \\
\text { not perform better than OB children in } 1 / 2 \text { LOC (jumping in } \\
\text { place), OC (dribbling ball), or balance (walking forward on a line) } \\
\text { skills; (iv) HW children did not perform better in most of the fine } \\
\text { motor tasks. }\end{array}$ \\
\hline Hardy et al. [37] & $\begin{array}{c}\frac{1962 \mathrm{~F}}{1962 \mathrm{M}} \\
(\sim 7-\sim 11 \mathrm{yrs})\end{array}$ & $\begin{array}{c}\text { POC applied to "Get Skilled:Get Active" } \\
\text { program (LOC skills: sprint run, vertical } \\
\text { jump, side gallop, and leap; } 3 \text { OC skills: } \\
\text { catch, over-arm throw, and kick): LOC and } \\
\text { OC scores }\end{array}$ & $\mathrm{n} / \mathrm{a}$ & $\mathrm{n} / \mathrm{a}$ & BMI & $\begin{array}{l}\text { (i) For LOC skills, there was a strong association between low } \\
\text { competency (low score) and OW/OB status, and this association } \\
\text { was consistent for most of the individual LOC skills; (ii) for OC } \\
\text { skills, no association was found with low competency in either M } \\
\text { or F children at } 7-11 \text { yrs, except for M aged } \sim 9 \text { yrs. }\end{array}$ \\
\hline Kelly et al. [38] & $\frac{216 \mathrm{M}}{(6-12 \mathrm{yrs})}$ & $\begin{array}{l}\text { TGMD-3 (LOC skills such as run, gallop, } \\
\text { hop, leap, jump, and slide, and OC skills } \\
\text { such as strike, dribble, catch, kick, throw, } \\
\text { and underhand roll) and "Get Skilled:Get } \\
\text { Active" and "Victorian FMS manual " } \\
\text { protocols (vertical jump and single leg } \\
\text { stance): LOC and OC scores, and total } \\
\text { gross motor score }\end{array}$ & $\mathrm{n} / \mathrm{a}$ & $\mathrm{n} / \mathrm{a}$ & BMI & $\begin{array}{c}\text { (i) HW children performed better in } 5 / 7 \text { LOC skills }(p<0.001 \text { ), } \\
\text { only } 3 / 8 \text { OC skills }(p=0.03 \text { ), and gross motor score }(p<0.001) \text { than } \\
\text { OW peers; (ii) no difference was observed in stability skills } \\
\text { (i.e., balance); (iii) children at } \sim 6.5 \text { yrs had significantly lower OC } \\
\text { scores than older peers (up to } ~ 11.5 \text {, with a peak score of OC at } \\
9.5 \text { yrs; (iv) M children were superior in total gross motor score } \\
\text { and OC score to F children. }\end{array}$ \\
\hline Laukkanen et al. [48] & $\begin{array}{c}1610 \mathrm{~F} 1655 \mathrm{M} \\
(6-9 \mathrm{yrs})\end{array}$ & $\mathrm{n} / \mathrm{a}$ & $\begin{array}{l}\text { KTK (balancing backward, jumping } \\
\text { laterally, hopping one leg, and shifting } \\
\text { platforms): total MQ score }\end{array}$ & $\mathrm{n} / \mathrm{a}$ & $\begin{array}{c}\text { BMI } \\
\text { percentile }\end{array}$ & $\begin{array}{l}\text { (i) BMIpercentile was negatively associated with } \mathrm{MQ}(\mathrm{r}=-0.131< \\
\mathrm{r}<-0.316 \text { ) in all European regions (northern, central and } \\
\text { southern); (ii) OW or OB children performed worse than } \mathrm{HW} \\
\text { peers from 5-92\% to } 12.07 \% \text { across northern, central, and southern } \\
\text { European regions; (iii) F children performed significantly lower } \\
\text { than M children (from } 2.36 \% \text { to } 9.03 \% \text { ) across the three regions. }\end{array}$ \\
\hline Lima et al. [49] & $\begin{array}{c}619 \mathrm{~F} \\
692 \mathrm{M} \\
(6-9 \mathrm{yrs}) \\
\end{array}$ & $\mathrm{n} / \mathrm{a}$ & $\begin{array}{l}\text { KTK (balancing backward, jumping } \\
\text { laterally, hopping one leg, and shifting } \\
\text { platforms): total MQ score }\end{array}$ & $\mathrm{n} / \mathrm{a}$ & S4SF & $\begin{array}{l}\text { (i) For both M and F children, S4SF had a strong association in the } \\
\text { development of AMC (by MQ score); (ii) higher S4SF levels at } 6 \text { yrs } \\
\text { (baseline) were associated with lower total scores across time (from } \\
6 \text { to } 9 \text { yrs); (iii) for M, the association became stronger even after } 9 \\
\text { yrs, while for F it tended to increase only from } 6 \text { to } 9 \text { yrs. }\end{array}$ \\
\hline Lopes et al. [50] & $\begin{array}{c}1826 \mathrm{~F} \\
1912 \mathrm{M} \\
\text { (6 to } 10 \mathrm{yrs}) \\
\end{array}$ & $\mathrm{n} / \mathrm{a}$ & $\begin{array}{l}\text { KTK (balancing backward, jumping } \\
\text { laterally, hopping one leg, and shifting } \\
\text { platforms): total and individual MQ } \\
\text { test score }\end{array}$ & $\mathrm{n} / \mathrm{a}$ & BMI & $\begin{array}{l}\text { (i) MQ was, on average, significantly higher in } \mathrm{M} \text { than in } \mathrm{F} \\
\text { children; (ii) curvilinear relationships between BMI and MQ were } \\
\text { indicated in F children, especially at } 10 \text { yrs; (iii) curvilinear } \\
\text { relationships were quite similar in M children at all ages; (iv) an } \\
\text { inverted parabolic relationship was found between individual } \\
\text { (LOC and stability skills) test scores. }\end{array}$ \\
\hline Lopes et al. [51] & $\begin{array}{c}281 \mathrm{~F} \\
315 \mathrm{M} \\
(9-12 \mathrm{yrs})\end{array}$ & $\mathrm{n} / \mathrm{a}$ & $\begin{array}{l}\text { KTK (balancing backward, jumping } \\
\text { laterally, hopping one leg, and shifting } \\
\text { platforms): total MQ score }\end{array}$ & $\mathrm{n} / \mathrm{a}$ & $\begin{array}{l}\text { BMI, FM, } \\
\text { and WC }\end{array}$ & $\begin{array}{l}\text { BMI, FM, and WC were negatively and significantly associated } \\
\text { with MQ in both sexes. }\end{array}$ \\
\hline
\end{tabular}


Table 1. Cont.

\begin{tabular}{|c|c|c|c|c|c|c|}
\hline \multirow{2}{*}{ Author } & \multirow{2}{*}{ Subjects } & \multicolumn{2}{|c|}{ AMC Measure } & \multirow{2}{*}{ PMC Measure } & \multirow{2}{*}{$\begin{array}{c}\text { Weight } \\
\text { Status } \\
\text { Measure }\end{array}$} & \multirow{2}{*}{ Results } \\
\hline & & Process-Oriented & Product-Oriented & & & \\
\hline Marmeleira et al. [11] & $\begin{array}{c}70 \mathrm{~F} \\
86 \mathrm{M} \\
\text { (6 to } 10 \mathrm{yrs}) \\
\end{array}$ & $\mathrm{n} / \mathrm{a}$ & $\begin{array}{l}\text { BOTMP-SF ( } 6 \text { subtests of gross motor } \\
\text { skills, } 6 \text { subtests of fine motor skills, and } \\
2 \text { subtests of both gross and fine motor } \\
\text { skills): total motor proficieincy standard } \\
\text { score and gross and fine motor } \\
\text { skill scores }\end{array}$ & $\mathrm{n} / \mathrm{a}$ & FM & $\begin{array}{l}\text { (i) FM was negatively associated with the gross and fine motor } \\
\text { composite score; (ii) FM was not associated with performance in } \\
\text { fine motor tasks, except for two tasks: drawing a line through a } \\
\text { straight path and sorting shape cards with preferred hand; (iii) for } \\
\text { both F and M children, FM was negatively associated with each } \\
\text { gross motor skill, while it showed a small negative association for } \\
\text { fine skills only in M children. }\end{array}$ \\
\hline Morrison et al. [52] & $\begin{array}{c}926 \mathrm{~F} \\
955 \mathrm{M} \\
(8-11 \mathrm{yrs})\end{array}$ & $\mathrm{n} / \mathrm{a}$ & $\begin{array}{l}\text { BOTMP-SF ( } 6 \text { subtests of gross motor } \\
\text { skills, } 6 \text { subtests of fine motor skills, } 2 \\
\text { subtests of both gross and fine motor } \\
\text { skills): total motor proficiency } \\
\text { standard score }\end{array}$ & $\begin{array}{l}\text { Self-Perception } \\
\text { Profile for Children: } \\
\text { PMC subscale score }\end{array}$ & BMI & $\begin{array}{l}\text { (i) An inverse relationship was found between BMI and total motor } \\
\text { proficiency standard score }(p<0.001) \text {; (ii) a positive relationship } \\
\text { was found between PMC and total motor proficiency standard } \\
\text { score for both M and F children; (iii) except for M children, BMI } \\
\text { was inversely }(p<0.05) \text { correlated to PMC; (iv) differences were } \\
\text { observed in AMC and PMC between F and M children }(p<0.05) \text {. }\end{array}$ \\
\hline Poulsen et al. [7] & $\begin{array}{c}59 \mathrm{~F} \\
57 \mathrm{M} \\
(6-11 \mathrm{yrs}) \\
\end{array}$ & $\mathrm{n} / \mathrm{a}$ & $\begin{array}{l}\text { BOTMP-2 (only } 5 \text { subtests of gross motor } \\
\text { skills: bilateral coordination, upper limb } \\
\text { coordination, balance, running speed } \\
\text { and agility, and strength): total and } \\
\text { individual motor proficiency } \\
\text { standard score }\end{array}$ & $\begin{array}{l}\text { The Self-Description } \\
\text { Questionnaire (physical } \\
\text { ability self-concept and } \\
\text { physical appearance } \\
\text { self-concept subscales) }\end{array}$ & BMI & $\begin{array}{l}\text { (i) OW children exhibited lower scores on all gross motor subtests } \\
\text { compared with HW peers }(p<0.05) \text {; (ii) OW children scored lower } \\
\text { on tests of self-concept perceptions of physical ability than HW } \\
\text { peers }(p<0.001) ; \text {; (iii) no relationships were found between BMI } \\
\text { and physical ability or physical appearance subscale scores; } \\
\text { (iv) BMI was inversely related with balance }(p<0.01 \text { ), strength } \\
\text { (p<0.001), running speed and agility }(p<0.001) \text {, and bilateral } \\
\text { coordination }(p<0.01) \text {. }\end{array}$ \\
\hline Rodrigues et al. [53] & \begin{tabular}{|c|}
$233 \mathrm{~F}$ \\
$239 \mathrm{M}$ \\
$\begin{array}{c}\text { from } 1 \text { st } \\
\text { grade }(\sim 6 \text { yrs }) \\
\text { to 4th grade }\end{array}$ \\
\end{tabular} & $\mathrm{n} / \mathrm{a}$ & $\begin{array}{l}\text { LOC skill (standing long jump), speed } \\
(50 \mathrm{~m} \text { dash), and agility (10 m shuttle } \\
\text { run) divided by low, average, and high } \\
\text { rates of change in motor competence }\end{array}$ & $\mathrm{n} / \mathrm{a}$ & BMI & $\begin{array}{l}\text { (i) Children in the low rate of change group exhibited negative or } \\
\text { very limited positive increases in motor competence levels from } \\
\text { 1st to } 4 \text { th grade; (ii) for LOC skills, children with low rates of } \\
\text { change over time presented an increased risk of OW/OB status. }\end{array}$ \\
\hline Spessato et al. [39] & $\begin{array}{c}96 \mathrm{~F} \\
82 \mathrm{M} \\
(4-7 \mathrm{yrs}) \text { of } \\
\text { which } \mathrm{n}=40 \\
(6 \mathrm{yrs}) \text { and } \\
\mathrm{n}=32(7 \mathrm{yrs})\end{array}$ & $\begin{array}{l}\text { TGMD-2 (LOC skills such as run, gallop, } \\
\text { hop, leap, jump, and slide, and OC skills } \\
\text { such as strike, dribble, catch, kick, throw, } \\
\text { and underhand roll): TGMD-2 scores. }\end{array}$ & $\mathrm{n} / \mathrm{a}$ & $\begin{array}{l}\text { Pictorial scale of } \\
\text { perceived competence } \\
\text { and Social Acceptance } \\
\text { (Physical competence } \\
\text { subscale): PMC score }\end{array}$ & BMI & $\begin{array}{c}\text { (i) TGMD-2 total score was inversely correlated with BMI for both } \\
6 \text { yrs and } 7 \text { yrs old; (ii) BMI was negatively correlated with PMC } \\
\text { score }(p<0.05) \text {; (iii) a small positive significant }(\mathrm{p}<0.05) \\
\text { correlation was found between TGMD-2 total score and PMC score } \\
\text { for } 6 \text { yr old children. }\end{array}$ \\
\hline Valentini et al. [21] & $\begin{array}{c}75 \mathrm{~F} \\
75 \mathrm{M} \\
(6-10 \mathrm{yrs})\end{array}$ & $\begin{array}{l}\text { TGMD-2 (LOC skills such as run, gallop, } \\
\text { hop, leap, jump, and slide, and OC skills } \\
\text { such as strike, dribble, catch, kick, throw, } \\
\text { and underhand roll): LOC and OC } \\
\text { subtest scores. }\end{array}$ & $\mathrm{n} / \mathrm{a}$ & $\begin{array}{l}\text { Pictorial scale of } \\
\text { perceived competence } \\
\text { and Self-Perception } \\
\text { Profile for Children: } \\
\text { row scores }\end{array}$ & BMI & $\begin{array}{l}\text { (i) BMI was inversely associated with LOC skills }(p<0.05) \text {; (ii) no } \\
\text { association was found between BMI and OC skills }(p>0.05) \text {; } \\
\text { (iii) an inverse relationship was found between PMC raw scores } \\
\text { and BMI }(p<0.05) \text {; (iv) PMC raw scores were also inversely } \\
\text { (p<0.05) related to LOC skills, while no significant relationship } \\
\text { was found with OC skills. }\end{array}$ \\
\hline
\end{tabular}

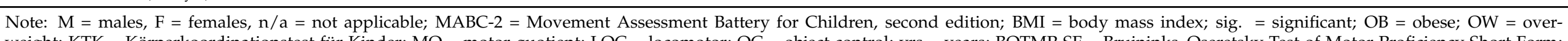

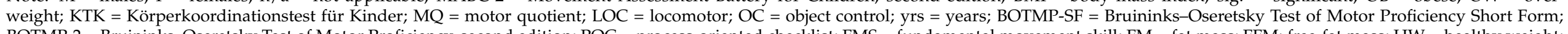

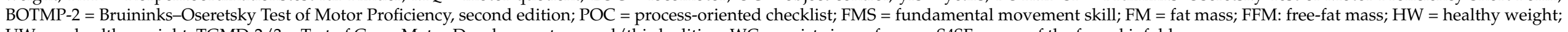
UW = unhealthy weight; TGMD-2/3 = Test of Gross Motor Development, second/third edition; WC = waist circumference; S4SF = sum of the four skinfolds. 


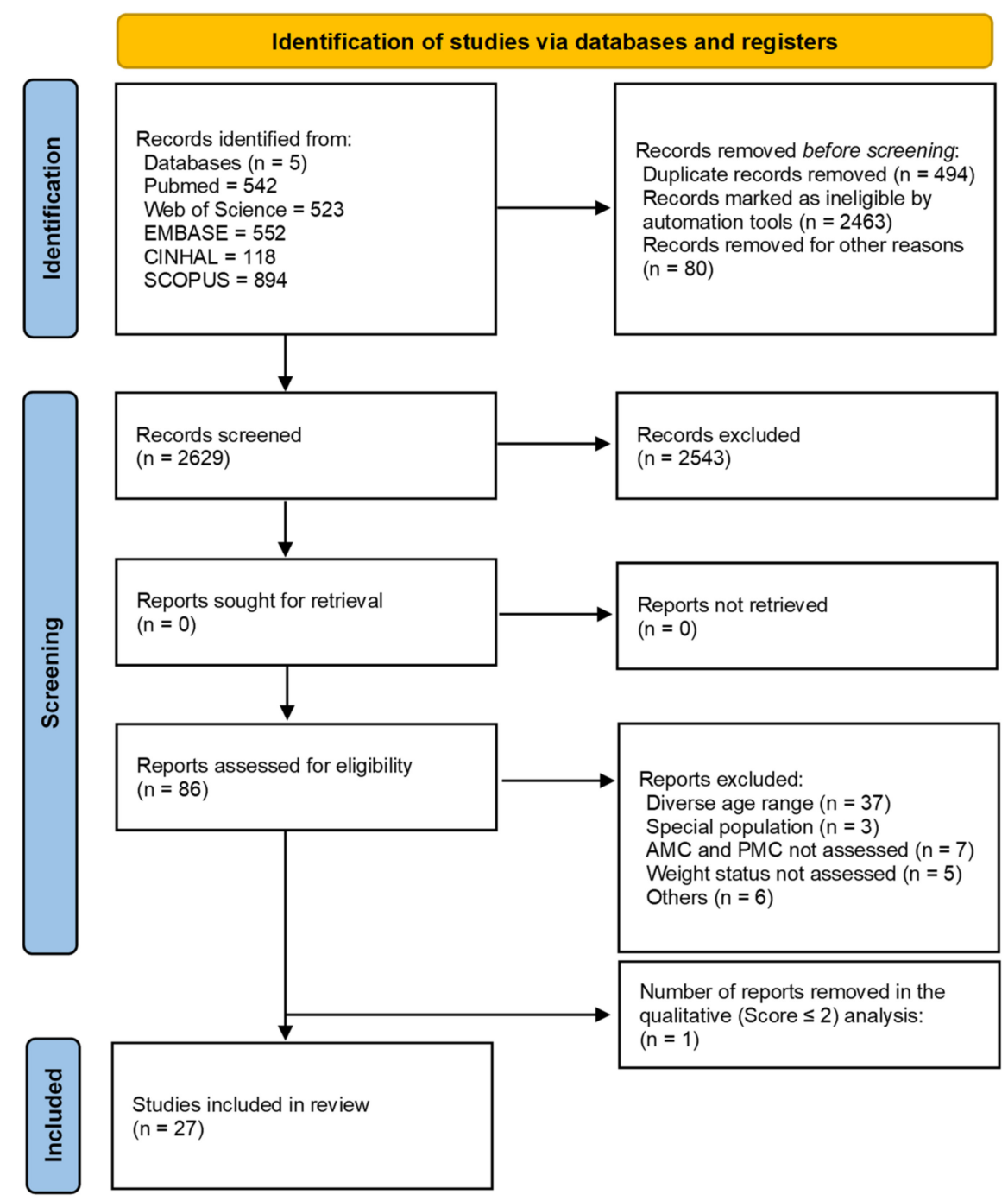

Figure 1. Flowchart of the review process. AMC = actual motor competence, $\mathrm{PMC}=$ perceived motor competence.

Most of the studies (26/27,96\%) used BMI or derived measures (BMIpercentile and BMI z-scores), alone or combined with waist circumference (WC) and fat mass (FM), to determine weight status. One study used the sum of the four skinfolds (S4SF). Two studies used only FM and free-fat mass (FFM), respectively. Overall, nine [30-36,38,39] and fifteen $[7,15,40-48,50-53]$ studies provided support for inverse associations between process- and product-oriented AMC assessments and BMI, respectively. The study of Comeau et al. [33] was the only study showing both a significant association between process-oriented AMC assessment, based on the Passport for Life program, and a nonsignificant association between process-oriented AMC assessment, based on the PLAYbasic program, and BMI. Three [31,33,35] and five studies [11,40,44,46,50] provided support for inverse associations between process- and product-oriented AMC assessment and FM. The study of Comeau et al. [33] was the only study showing both a significant association between process-oriented AMC assessment, based on the PLAYbasic program, and a nonsignificant association between process-oriented AMC assessment, based on the Passport for Life program, and WC. Two studies [40,50] provided support for a significant inverse association between product-oriented AMC assessment and WC. One study [53] showed a significant inverse relationship between product-oriented AMC assessment and S4SF. One study [44] showed a significant positive relationship between product-oriented AMC assessment and FFM (see Table 1). 
For AMC, twenty-one studies included a total score within their analysis, providing overall support for an inverse association of total scores of process- [31-36,39] and productoriented [7,15,40-43,45,46,48-52] assessment and BMI. Fourteen studies [7,21,30,32,33,35,38, $40,42,44,45,47,50,53$ ] also employed individual subtest scores (locomotor, object control, and stability skills) accompanied with a measure of BMI. Specifically, twelve studies indicated an inverse association between locomotor skills and BMI [7,21,30,32,33,35,38,44,45,47,50,53], overall reporting different levels of skill between children with healthy and unhealthyweight (overweight or obese) status; six each for process- [21,30,32,33,35,38] and productoriented $[7,44,45,47,50,53]$ assessments. Only one study provided support for an inverse association between object control skills and BMI, as measured in both both process- [32] and product-oriented [42] assessments. Only ten studies reported clear outcomes based on stability skills in relation to BMI. Five studies $[7,40,44,45,50]$ indicated an inverse association between stability and BMI only for product-oriented assessments, while one study [15] did not find an association. Likewise, two studies [33,38] employing process-oriented assessments did not provide support for the aforementioned association. Finally, in two studies, it was not possible to infer a clear association, not even in healthy- and unhealthyweight (overweight or obese) children (see Table 1).

For PMC, four studies [21,34,39,52] provided support for an inverse association with BMI, while only one study [7] did not show a significant association. Specifically, Morrison et al. [52] showed a significantly inverse relationship between PMC and BMI only for F children, while no significant association was observed in M children (see Table 1).

Nine studies [11,30,34,38,41,45,48,50,52] reported differences by gender. Seven studies $[30,38,41,45,48,50,52]$ found superior AMC in M compared to F children. Specifically, five studies $[38,41,48,50,52]$ reported higher total scores in product- and process-oriented assessments, and two studies [30,38] reported better performance in object-control skills (e.g., kick and throw) in $\mathrm{M}$ than in F children. One study [45] reported better performance in locomotor skills (jumping and shifting) in $\mathrm{M}$ than in F children and a better performance in stability skills in F than in M children. One study [11] showed a significant relationship between FM and fine motor skills only in M children. Two studies [34,52] reported higher $\mathrm{PMC}$ in $\mathrm{M}$ than in $\mathrm{F}$ children.

Figure 2 shows a schematic representation of AMC (total and subtest scores by processand product-oriented assessments) and PMC outcomes in relation to weight status (BMI) derived from quantitative data interpretation. In regard to total score, most of the studies indicated that AMC and PMC were higher in healthy-weight children compared with unhealthy-weight peers, regardless of the type of AMC assessment. Likewise, healthyweight children presented higher locomotor subtest scores compared with unhealthy peers. Regarding object control subtest score, most of the studies did not find differences in relation to weight status, also regardless of the type of AMC assessment. Conversely, healthy-weight children presented higher stability subtest scores based on product-oriented rather than process-oriented assessments.

Figure 3 shows a schematic representation of AMC (total and subtest score) and PMC outcomes in relation to gender (M and F) derived from quantitative data interpretation. Most of the studies indicated that M outperformed F children on AMC and PMC total scores, and on locomotor and object control subtest scores, while F children appear superior in stability subtest scores to M peers. 


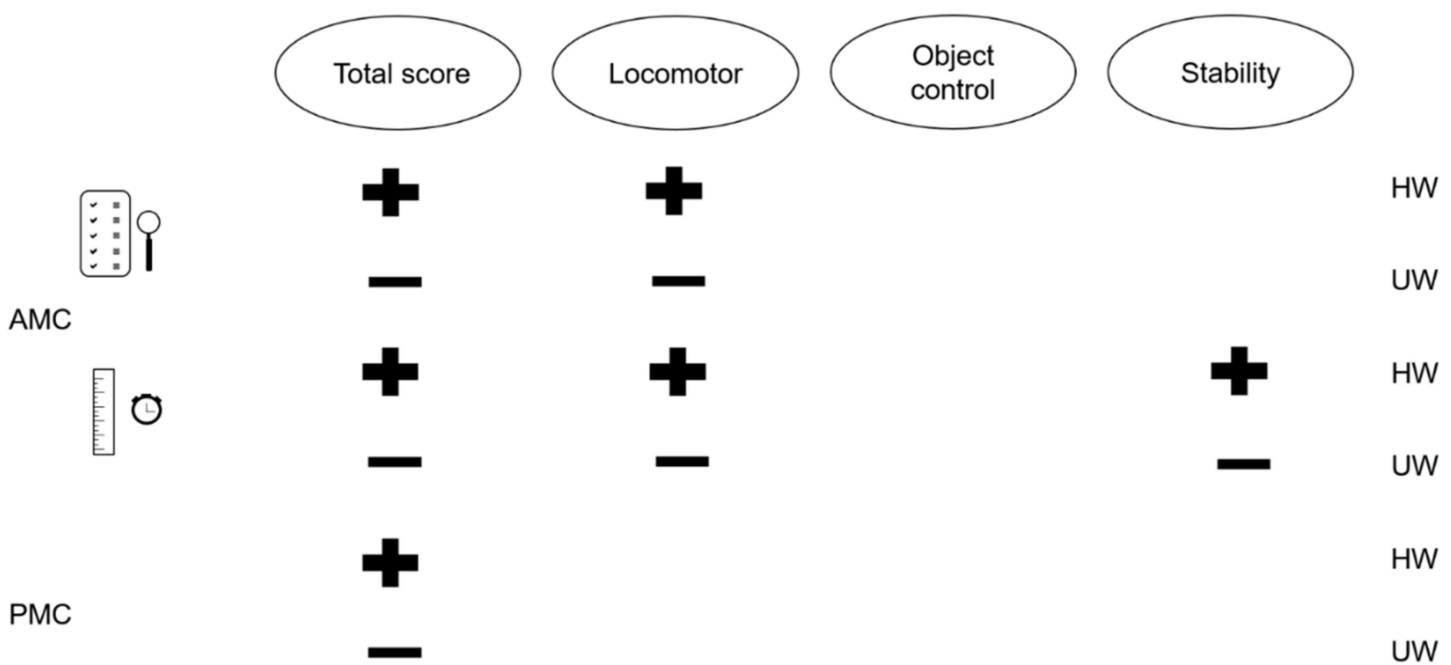

Figure 2. Schematic representation of both actual (AMC) and perceived (PMC) motor competence in relation to weight status based on body mass index. The checklist and magnifying glass refer to qualitative assessments based on process-oriented tests, while the ruler and stopwatch refer to quantitative assessments based on product-oriented tests. The symbols indicate whether a corresponding outcome was linked to AMC and PMC in favor (plus) or not (minus) of healthy-weight (HW) and unhealthy-weight (UW) status.
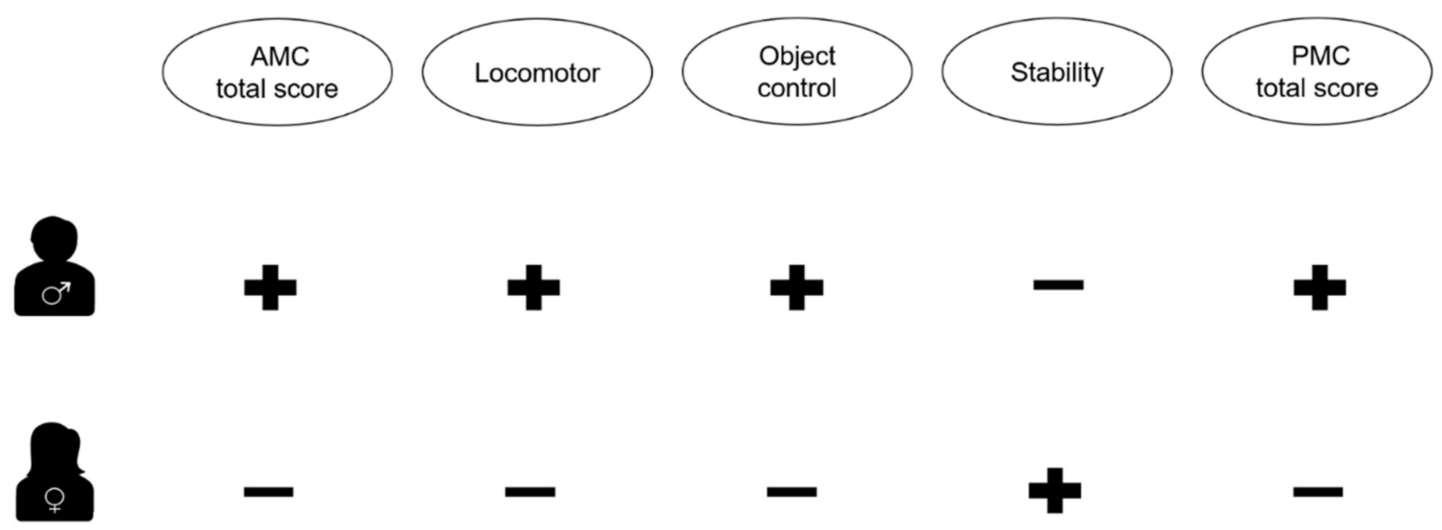

Figure 3. Schematic representation of both actual (AMC) and perceived (PMC) motor competence mediated by gender. The symbols indicate whether a corresponding outcome was linked to AMC and PMC in favor (plus) or not (minus) of male and female sex.

\subsection{Reporting Bias Assessment}

The overall results from the risk of bias assessment are reported in Table 2. Three studies were classified as having low risk of bias (high quality studies) [32,42,46], twentythree studies were classified as having medium risk of bias $[7,11,15,21,30,31,33-36,38-41,43-$ 45,47-53], and one study was classified as having high risk of bias [37]. The latter study was excluded from the analysis. Fourteen studies (51\%) sufficiently described the participant eligibility criteria. Eight studies (29.5\%) clearly randomized their sample selection. Most of the studies (92.5-96\%) reported the sources and details of methods and AMC or PMC assessments used, also including acceptable reliability for the specific age group. Only one study $(96 \%)$ reported a power calculation. 
Table 2. Study quality checklist and corresponding quality score for each of the selected study.

\begin{tabular}{|c|c|c|c|c|c|c|c|}
\hline Study & $\begin{array}{l}\text { Did the Study } \\
\text { Describe the } \\
\text { Participant } \\
\text { Eligibility } \\
\text { Criteria? }\end{array}$ & $\begin{array}{l}\text { Were the } \\
\text { Participants } \\
\text { Randomly } \\
\text { Selected? }\end{array}$ & $\begin{array}{l}\text { Did the Study Report the } \\
\text { Sources and Details of the } \\
\text { AMC or PMC Assessment } \\
\text { Used, and Did the } \\
\text { Instrument Have Acceptable } \\
\text { Reliability for the Specific } \\
\text { Age Group? }\end{array}$ & $\begin{array}{l}\text { Did the Study Report the } \\
\text { Sources and Details of the } \\
\text { Assessments of Potential } \\
\text { Benefits, and Did All of the } \\
\text { Methods Have Acceptable } \\
\text { Reliability for the Specific } \\
\text { Age Group? }\end{array}$ & $\begin{array}{l}\text { Did the Study Report a } \\
\text { Power Calculation, } \\
\text { and Was the Study } \\
\text { Adequately Powered } \\
\text { to Detect } \\
\text { Hypothesized } \\
\text { Relationships? }\end{array}$ & $\begin{array}{l}\text { Did the Study Report } \\
\text { the Numbers of } \\
\text { Individuals who } \\
\text { Completed Each of the } \\
\text { Different Measures, } \\
\text { and Did Participants } \\
\text { Complete at Least } 80 \% \\
\text { of the AMC or PMC } \\
\text { and Benefit Measures? }\end{array}$ & $\begin{array}{l}\text { Quality Score } \\
\text { (Max 6) }\end{array}$ \\
\hline Augustijn et al. [40] & 0 & 0 & 1 & 1 & 0 & 1 & 3 \\
\hline Bryant et al. [30] & 1 & 0 & 1 & 1 & 0 & 1 & 4 \\
\hline Bryant et al. [31] & 1 & 0 & 1 & 1 & 0 & 1 & 4 \\
\hline Cliff et al. [32] & 1 & 1 & 1 & 1 & 0 & 1 & 5 \\
\hline Cheng et al. [41] & 1 & 0 & 1 & 1 & 0 & 1 & 4 \\
\hline Chowdhury et al. [42] & 1 & 1 & 1 & 1 & 0 & 1 & 5 \\
\hline Comeau et al. [33] & 0 & 1 & 1 & 1 & 0 & 1 & 4 \\
\hline Coppens et al. [43] & 0 & 1 & 1 & 1 & 0 & 0 & 3 \\
\hline De Meester et al. [34] & 1 & 0 & 1 & 1 & 0 & 1 & 4 \\
\hline Dos'Santos et al. [44] & 0 & 0 & 1 & 1 & 0 & 1 & 3 \\
\hline D'Hondt et al. [45] & 0 & 1 & 1 & 1 & 0 & 1 & 4 \\
\hline D'Hondt et al. [46] & 1 & 1 & 1 & 1 & 0 & 1 & 5 \\
\hline D'Hondt et al. [47] & 1 & 0 & 1 & 1 & 0 & 1 & 4 \\
\hline Duncan and Stanley [36] & 0 & 0 & 1 & 1 & 0 & 1 & 3 \\
\hline Gentier et al. [15] & 1 & 0 & 1 & 1 & 0 & 1 & 4 \\
\hline Hardy et al. [37] & 0 & 0 & 0 & 1 & 0 & 1 & 2 \\
\hline Kelly et al. [38] & 0 & 0 & 1 & 1 & 1 & 1 & 4 \\
\hline Laukkanen et al. [48] & 0 & 1 & 1 & 1 & 0 & 1 & 4 \\
\hline Lima et al. [49] & 0 & 0 & 1 & 1 & 0 & 1 & 3 \\
\hline Lopes et al. [50] & 0 & 0 & 1 & 1 & 0 & 1 & 3 \\
\hline Lopes et al. [51] & 1 & 0 & 0 & 1 & 0 & 1 & 3 \\
\hline Marmeleira et al. [11] & 0 & 0 & 1 & 1 & 0 & 1 & 3 \\
\hline Morrison et al. [52] & 1 & 0 & 1 & 1 & 0 & 1 & 4 \\
\hline Poulsen et al. [7] & 1 & 0 & 1 & 1 & 0 & 0 & 3 \\
\hline Rodrigues et al. [53] & 1 & 0 & 1 & 0 & 0 & 1 & 3 \\
\hline Spessato et al. [39] & 0 & 0 & 1 & 1 & 0 & 1 & 3 \\
\hline Valentini et al. [21] & 0 & 1 & 1 & 1 & 0 & 1 & 4 \\
\hline
\end{tabular}

Note: $\mathrm{AMC}=$ actual motor competence; $\mathrm{PMC}=$ perceived motor competence. 
Tables 3-5 summarize the level of scientific evidence linked to the assessed risk of bias. Regarding AMC based on process-oriented performance, of the studies providing support for an association with BMI, only one was deemed to have low risk of bias [32]. Given the established criteria, the level of evidence remains uncertain. Of the studies providing support for an association between AMC based on process-oriented performance and FM and WC, none were deemed to have low risk of bias. Given the established criteria, the level of scientific evidence remains lacking. Regarding AMC based on product-oriented performance, of the studies providing support for an association with BMI, only two [42,46] were deemed to have low risk of bias, while of those providing support for an association with FM, only one [46] was deemed to have low risk of bias. Given the established criteria, the level of evidence remains uncertain. In regard to WC, S4SF, and FFM, no studies were deemed to have low risk of bias; thus, the level of scientific evidence remains lacking. In regard to PMC assessment, of the studies providing support for an association with BMI, none were deemed to have low risk of bias. Given the established criteria, the level of scientific evidence remains lacking.

Table 3. Summary of the studies providing support for the association between process-oriented AMC and weight status.

\begin{tabular}{|c|c|c|c|c|c|c|}
\hline \multirow{3}{*}{ Weight Status } & \multirow{2}{*}{\multicolumn{2}{|c|}{$\begin{array}{c}\text { Associated with AMC } \\
\text { Process }\end{array}$}} & \multirow{3}{*}{$\begin{array}{c}\begin{array}{c}\text { Not } \\
\text { Associated } \\
\text { with AMC }\end{array} \\
\text { Reference }\end{array}$} & \multirow{3}{*}{$\begin{array}{c}\begin{array}{c}\text { Summary } \\
\text { Coding }\end{array} \\
\mathbf{n} / \mathbf{N} \\
\text { for Benefit }\end{array}$} & \multirow{3}{*}{$\begin{array}{c}\text { Studies of } \\
\text { Low Risk } \\
\text { of Bias }\end{array}$} & \multirow{3}{*}{$\begin{array}{l}\text { Level of } \\
\text { Evidence }\end{array}$} \\
\hline & & & & & & \\
\hline & Reference & Association & & & & \\
\hline $\begin{array}{l}\text { BMI, BMI z-scores, } \\
\text { and BMIpercentile }\end{array}$ & $\begin{array}{c}{[30]^{\mathrm{a}},[31],[32]^{\mathrm{b}},} \\
{[33]^{\mathrm{c}},[34],[35],} \\
{[36],[38],[39]^{\mathrm{a}}}\end{array}$ & inverse & {$[34]^{\mathrm{d}}$} & $9 / 10(90 \%)$ & $1 / 10$ & $?$ \\
\hline $\mathrm{FM}$ & {$[32],[34]^{\mathrm{c}, \mathrm{d}},[36]$} & inverse & $\mathrm{n} / \mathrm{a}$ & $3 / 3(100 \%)$ & $0 / 3$ & $x$ \\
\hline WC & {$[34]^{\mathrm{d}}$} & inverse & {$[34]^{\mathrm{c}}$} & $1 / 2(50 \%)$ & $0 / 2$ & $x$ \\
\hline S4SF & $\mathrm{n} / \mathrm{a}$ & $\mathrm{n} / \mathrm{a}$ & $\mathrm{n} / \mathrm{a}$ & $\mathrm{n} / \mathrm{a}$ & $\mathrm{n} / \mathrm{a}$ & $\mathrm{n} / \mathrm{a}$ \\
\hline FFM & $\mathrm{n} / \mathrm{a}$ & $\mathrm{n} / \mathrm{a}$ & $\mathrm{n} / \mathrm{a}$ & $\mathrm{n} / \mathrm{a}$ & $\mathrm{n} / \mathrm{a}$ & $\mathrm{n} / \mathrm{a}$ \\
\hline
\end{tabular}

Note: $\mathrm{AMC}=$ actual motor competence; $\mathrm{PMC}$, perceived motor competence; $\mathrm{n} / \mathrm{a}=$ not applicable; $\mathrm{BMI}=$ body mass index; $\mathrm{FM}=$ fat mass; FFM: free-fat mass; $\mathrm{WC}=$ waist circumference; $\mathrm{S} 4 \mathrm{SF}=$ sum the four skinfolds. ${ }^{\mathrm{a}}=$ only for mastery level of locomotor skills; ${ }^{\mathrm{b}}=$ mastery level of both locomotor and object control skills; ${ }^{\mathrm{c}}=$ only for Passport for Life AMC tool assessment; ${ }^{\mathrm{d}}=$ only for gross motor skills; $\mathrm{d}=$ only for PLAYbasic AMC tool assessment; $\mathrm{n}=$ number of studies that report support for relationship; $\mathrm{N}=$ number of studies that examined and reported possible associations of AMC and PMC with weight status; ? = uncertain evidence; $x=$ lack of scientific evidence.

Table 4. Summary of the studies providing support for the association between product-oriented AMC and weight status.

\begin{tabular}{|c|c|c|c|c|c|c|}
\hline \multirow[b]{3}{*}{ Weight Status } & \multicolumn{2}{|c|}{ Associated with AMC } & \multirow{3}{*}{$\begin{array}{c}\begin{array}{c}\text { Not } \\
\text { Associated } \\
\text { with AMC }\end{array} \\
\text { Reference }\end{array}$} & \multirow{3}{*}{$\begin{array}{c}\begin{array}{c}\text { Summary } \\
\text { Coding }\end{array} \\
\begin{array}{c}\mathbf{n} / \mathbf{N} \\
\text { for Benefit }\end{array}\end{array}$} & \multirow{3}{*}{$\begin{array}{c}\text { Studies of } \\
\text { Low Risk } \\
\text { of Bias }\end{array}$} & \multirow{3}{*}{$\begin{array}{l}\text { Level of } \\
\text { Evidence }\end{array}$} \\
\hline & \multicolumn{2}{|c|}{ Product } & & & & \\
\hline & Reference & Association & & & & \\
\hline $\begin{array}{l}\text { BMI, BMI z-scores, } \\
\text { and BMIpercentile }\end{array}$ & {$[7,15,40-48,50-53]$} & inverse & $\mathrm{n} / \mathrm{a}$ & $15 / 15(100 \%)$ & $2 / 15$ & $?$ \\
\hline FM & {$[11,40,44,46,51]$} & inverse & $\mathrm{n} / \mathrm{a}$ & $5 / 5(100 \%)$ & $1 / 5$ & $?$ \\
\hline WC & {$[40,51]$} & inverse & $\mathrm{n} / \mathrm{a}$ & $2 / 2(100 \%)$ & $0 / 2$ & $x$ \\
\hline S4SF & [49] & inverse & $\mathrm{n} / \mathrm{a}$ & $1 / 1(100 \%)$ & $0 / 1$ & $x$ \\
\hline FFM & {$[44]^{\mathrm{a}}$} & positive & $\mathrm{n} / \mathrm{a}$ & $1 / 1(100 \%)$ & $0 / 1$ & $x$ \\
\hline
\end{tabular}

Note: $\mathrm{AMC}=$ actual motor competence; $\mathrm{PMC}$, perceived motor competence; $\mathrm{n} / \mathrm{a}=$ not applicable; $\mathrm{BMI}=$ body mass index; $\mathrm{FM}=\mathrm{fat}$ mass; FFM: free-fat mass; $\mathrm{WC}=$ waist circumference; $\mathrm{S} 4 \mathrm{SF}=$ sum the four skinfolds. ${ }^{\mathrm{a}}=$ except for balancing backward; $\mathrm{n}=$ number of studies that report support for relationship; $\mathrm{N}=$ number of studies that examined and reported possible associations of AMC and PMC with weight status; ? = uncertain evidence; $\mathrm{x}=$ lack of scientific evidence. 
Table 5. Summary of the studies providing support for the association between PMC and weight status.

\begin{tabular}{|c|c|c|c|c|c|c|}
\hline \multirow{2}{*}{$\begin{array}{c}\text { PMC } \\
\text { Weight Status }\end{array}$} & \multicolumn{2}{|c|}{ Associated with PMC } & \multirow{2}{*}{$\begin{array}{c}\text { Not } \\
\text { Associated } \\
\text { with PMC }\end{array}$} & \multirow{2}{*}{$\begin{array}{c}\begin{array}{c}\text { Summary } \\
\text { Coding }\end{array} \\
\mathbf{n} / \mathbf{N} \\
\text { for Benefit }\end{array}$} & \multirow{2}{*}{$\begin{array}{c}\text { Studies of } \\
\text { Low Risk } \\
\text { of Bias }\end{array}$} & \multirow{2}{*}{$\begin{array}{l}\text { Level of } \\
\text { Evidence }\end{array}$} \\
\hline & Reference & Association & & & & \\
\hline $\begin{array}{l}\text { BMI, BMI z-scores, } \\
\text { and BMIpercentile }\end{array}$ & $\underset{\mathrm{a}}{[21],[34],[39],[52]}$ & inverse & [7] & $4 / 5(80 \%)$ & $0 / 5$ & $\mathrm{x}$ \\
\hline FM & $\mathrm{n} / \mathrm{a}$ & $\mathrm{n} / \mathrm{a}$ & $\mathrm{n} / \mathrm{a}$ & $\mathrm{n} / \mathrm{a}$ & $\mathrm{n} / \mathrm{a}$ & $\mathrm{n} / \mathrm{a}$ \\
\hline WC & $\mathrm{n} / \mathrm{a}$ & $\mathrm{n} / \mathrm{a}$ & $\mathrm{n} / \mathrm{a}$ & $\mathrm{n} / \mathrm{a}$ & $\mathrm{n} / \mathrm{a}$ & $\mathrm{n} / \mathrm{a}$ \\
\hline S4SF & $\mathrm{n} / \mathrm{a}$ & $\mathrm{n} / \mathrm{a}$ & $\mathrm{n} / \mathrm{a}$ & $\mathrm{n} / \mathrm{a}$ & $\mathrm{n} / \mathrm{a}$ & $\mathrm{n} / \mathrm{a}$ \\
\hline FFM & $\mathrm{n} / \mathrm{a}$ & $\mathrm{n} / \mathrm{a}$ & $\mathrm{n} / \mathrm{a}$ & $\mathrm{n} / \mathrm{a}$ & $\mathrm{n} / \mathrm{a}$ & $\mathrm{n} / \mathrm{a}$ \\
\hline
\end{tabular}

Note: PMC, perceived motor competence; $\mathrm{n} / \mathrm{a}=$ not applicable; BMI = body mass index; FM = fat mass; FFM: free-fat mass; WC = waist circumference; $\mathrm{S} 4 \mathrm{SF}=$ sum the four skinfolds. ${ }^{\mathrm{a}}=$ only for female; $\mathrm{n}=$ number of studies that report support for relationship; $\mathrm{N}=$ number of studies that examined and reported possible associations of AMC and PMC with weight status; $\mathrm{x}=$ lack of scientific evidence.

\section{Discussion}

The aim of the present study was to provide a synopsis of the current research investigating the relation of AMC and PMC with weight status, also in the context of potential gender differences. Overall, most of the studies reported an inverse association between AMC (process- and product-oriented assessment) and weight status (BMI and BMI-related variables, FM, WC, S4SF). This result applied also to PMC, for which an inverse association was found with BMI. However, according to the qualitative analysis (risk of bias assessment), the main findings revealed that the association between AMC and PMC with weight status remains unclear or lacking.

The present finding appears to be supported by a previous review on the relationship between AMC (i.e., fundamental movement skills) and weight status in children [18]. The authors found an inverse relationship between BMI and AMC, in terms of product- and process-oriented performance and locomotor, object-control, and stability skills, with the overall level of evidence still uncertain, which is in line with the present results. Of note, in most of the studies analyzed by both the present and Slotte and colleagues' reviews, weight status was primarily established by BMI as a sort of predictor for overweight and obese condition across childhood and adolescence. However, being derived from weight and height, BMI does not account for specific information on a child's body composition (i.e., adipose or muscle tissue); thus, further measures more closely linked to body composition should be employed to increase understanding of the relationship between AMC and weight status.

Accordingly, Slotte et al. [18] also reported studies providing support for the association between fundamental movement skills and WC, skinfolds, and FM. Of these, WC and FM were the only weight status variables significantly correlated with AMC that parallelly presented an overall strong level of evidence. Nevertheless, the present findings provided conflicting outcomes. Indeed, although we found a significant relationship of WC and FM (inverse) with AMC, the level of evidence remained lacking. This condition also applied for other variables such as S4SF (inverse) and FFM (positive). Beyond the diverse age range considered in the review of Slotte and colleagues (3-12 yrs), a related idea which might explain such an inconsistent result is the different approach adopted to assess FM within the selected studies. In the present review, eight studies employed FM as an inclusive [31,33,35,40,44,46,51] or exclusive [11] measure of weight status, which was derived by summing specific skinfolds $[11,31,35,44]$ and by analyzing bioelectrical impedance $[33,40,46,51]$, whereas Slotte et al. [18] reported that only one study, which derived it from dual-energy X-Ray absorptiometry, used FM. On one hand, including a measure of body composition such as FM may provide more precise evidence on the interplay between AMC and weight status $[18,29,54]$. On the other hand, the number of various approaches to determine weight status along with the limited number of existing 
studies (in terms of quality and quantity) do not provide sufficient support for a better understanding of the association between AMC and weight status.

Given the state of the evidence, a cursory glance at Table 1 and Figure 2 reveals information potentially useful for understanding the association between AMC and weight status (overall determined by BMI) on an individual level. AMC performance appeared broadly lower in overweight (OW) and obese (OB) children compared with healthy-weight (HW) peers. Among the studies employing process-oriented AMC assessments, a significant inverse association was found between overall AMC score, assessed by TGMD-2 [34,39], TGMD-3 [38], and fundamental movement screen tests [36], and BMI. Process-oriented assessments test how a motor skill is accomplished [55]. From this approach, qualitative information may be obtained to better infer whether a skill criterion (or mastery level) is present or not. Accordingly, HW children (6-10 yrs) showed a higher mastery level for locomotor (i.e., run, gallop, hop, leap, jump, and slide) and object-control (i.e., strike, dribble, catch, kick, throw, and underhand roll) skills than their overweight and obese peers [32]. Apparently, an unhealthy-weight condition (e.g., a high BMI indicating overweight or obesity) alters the way a child can move because of the negative interaction between large body mass and gravity. Bryant et al. [30] revealed that such an alteration becomes more accentuated for those skills requiring moving the whole body (e.g., sprint run) compared with those involving one side of the body (e.g., kick) during childhood. In this vein, while children (6-11 yrs) classed as having low locomotor skill (e.g., sprint, hop and gallop) exhibited significantly higher BMI or FM than those classed as having medium and high locomotor skill, no difference in BMI or FM was observed as a function of object control skill (e.g., catch, kick, overarm throw) mastery level [35]. Hence, the overall picture arising from these results would indicate a diverse interplay between locomotor and object control skill proficiency and weight status, for which the extent of the relationship can also vary (significant versus nonsignificant, respectively) in school-aged (6-10 yrs) children [21]. Notably, this consideration assumes relevance when bearing in mind that locomotor and object control skill levels can fall at different age periods during childhood. Indeed, locomotor score by "Move it Groove it" programs may drop at 8-9 yrs of age, while object control score may drop later (9-10 yrs) [30]. Although such information might become useful when aiming to develop the AMC level of school-aged children, the level of evidence remains unclear. Further qualitative studies are warranted to corroborate, or not, what the present review tentatively provided from the current literature.

Product-oriented assessments provide the outcome of a movement [56] regardless how the movement itself is performed. Specifically, outcomes are represented by quantitative scores, perhaps expressing running speed, jump distance, or number of successful attempts [56]. The studies employing product-oriented AMC assessments observed a significant inverse association between overall AMC score, as assessed by MABC-2 [40], BOTMP-SF [7,11,41,42,52] and BOTMP-2 [15], and KTK [43-49,51] tests, and weight status (i.e., $\mathrm{BMI}$ ), with $\mathrm{OW}$ and $\mathrm{OB}$ children demonstrating lower performance than their $\mathrm{HW}$ peers. Accordingly, Cheng et al. [41] found that OW and OB children exhibited lower gross motor score by BOTMP-SF test (based on locomotor, stability, and object control skills) than their HW counterparts at age 10 yrs. In the study by D'Hondt et al. [45], OW and OB children ranging from 10-12 yrs of age presented lower KTK scores on the locomotor (two-legged jumping) item. It was also reported that OW and OB children of 8-12 yrs of age presented lower stability (i.e., walking backwards) item scores than HW [45]. Likewise, a dominant finding from the study of Poulsen et al. [7] was the significant relationship between balance and BMI in children aged 6-11. However, although OB children scored globally lower than HW children in BOTMP-2, Gentier et al. [15] found no difference between $\mathrm{OB}$ and HW children in three items linked to a combination of locomotor and stability skills (bilateral coordination while jumping in place and walking forward) and object control (dribbling a ball). Moreover, for children ranging from 7 to 11 yrs of age, HW presented significantly higher locomotor and stability skills (i.e., one-board balance, heel-to-toe walking forward, and hopping on mats), while no differences were observed in 
object control skills (i.e., catching with two hands and throwing a beanbag onto mat). All together, these findings yielded additional data on the different AMC outcomes obtained when each different subtest (e.g., gross motor skills based on locomotor, object control, and stability) was considered. It appears that, compared with object control, locomotor skills are likely to better differentiate HW school-aged children from their OW and OB peers than either a quantitative (product) or qualitative (process) AMC screening. This would suggest that an unhealthy-weight status might affect locomotor skills to a greater extent compared with object control skills in children. However, the level of evidence is still uncertain. Additional high-quality studies will have to explore the association between different subtests of AMC with weight status to provide health professionals or educators with specific information about how to target interventions in school-aged children.

According to Stodden et al. [57], PMC may influence the extent of the association between AMC and physical activity, becoming stronger with increasing age within childhood. To the authors' knowledge, Khodaverdi et al. [58] were the first demonstrating a link between AMC, PMC, and physical activity in children (i.e., $8-9$ yrs), partially supporting the conceptual model of motor development proposed by Stodden and colleagues. Then, Utesch et al. [17] also provided support for this link in relatively matched-age children (9 yrs), while Barnett et al. [59] anticipated those findings in adolescent individuals (14.2 to 18.3 yrs). However, Hall et al., [60] showed that PMC had no mediating effects on AMC and physical activity in early-years-aged children (4-6 years). Cognitive capability linked to a child's own perception of their motor competence develops during the school years, sharpening the outcome between AMC and PMC. Accordingly, several studies showed a significant and positive relationship between AMC and PMC [34,39,52]. Notably, there is a synergetic role of AMC and PMC on young individuals' physical activity that can influence their weight status [45,58]. Indeed, a child's accurate self-perception of their own motor competence may foster their physical activity with a gradual increase of their $\mathrm{AMC}$, an augmented motivation to continue exercising, and a consequent BMI reduction $[17,21,34]$. In the present review, most of the analyzed studies observed a significant inverse relationship between PMC and BMI [21,34,39,52], while only one study did not report a significant association [7]. Although it seems that PMC plays a critical role, with regard to children's weight status [34], in children's pursuing an active lifestyle (continuing physical activity), the qualitative analysis based on risk of bias assessment proved a lack of evidence; even the low number of studies embedding a subjective evaluation of the motor ability or competence in school-age children hardly contribute to the understanding of the interplay between PMC and weight status. Additional research is needed to elucidate the underlying factors inherent to children's ability to perceive their own motor skills in relation to weight status [22].

The current review also provided overall data on AMC/PMC and weight status by gender difference, which are summed up in Figure 3. Most of the studies agreed that M children exhibit superior AMC to F children $[30,38,41,45,48,50,52,61]$ regardless the type of assessment (process or product). Among these studies, Bryant et al. [30] and Kelly et al. [38] agreed about the superior performance in object-control skills (e.g., kick and throw) by $\mathrm{M}$ compared with $\mathrm{F}$ children with ages ranging from 6 to 12 yrs. Moreover, Bryant et al. [30] found F children to have superior scores in balance skills compared to $\mathrm{M}$ children. These findings appear to be in line with those of previous studies conducted over early childhood [62] and adolescence [23]. Notably, the two former and latter studies employed process-oriented assessments (i.e., the "Move it Groove it", TGMD-2, TGMD-3, and "Get Skilled:Get Active" tests, respectively), while no studies (among those analyzed by this review) reported sex differences in object control skills assessed by product-oriented assessments. Rather, D'Hondt et al. [45] reported higher KTK scores for locomotor skills (jumping and shifting items) in $\mathrm{M}$ than in $\mathrm{F}$ children and better performance in stability skills (balancing) in $\mathrm{F}$ than in $\mathrm{M}$ children. Only one study reported a gender difference in PMC, in favor of M compared with F children [52]. Intuitively, this information may promote additional study in order to provide extended knowledge on gender differences in 
AMC and PMC in relation to weight status. Such research would help health professionals and educators to consider potential sex-specific intervention on locomotor, object control, and stability skills in order to promote psychomotor development in a more comprehensive way during the primary school period.

This systematic review had limitations that should be acknowledged. The massive use of BMI as a discriminating measure of weight status might impact the interpretation of the qualitative and quantitative data. Indeed, BMI does not provide detailed information on body composition. Perhaps future reviews will have to restrict their analysis to alternative approaches (e.g., bioelectrical impedance) to estimating body composition. However, to date, most studies have included BMI in their protocol, making the sole use of an alternative measure difficult within a systematic process of revision. Due to the low numbers of assessments used by the reviewed studies, fine motor skills were not included within the qualitative (and quantitative) analysis but were occasionally mentioned. Again, additional studies focusing on fine motor skills might contribute to enrich the understanding of the interplay between AMC and weight status through the systematic review approach.

\section{Practical Implications}

Besides the need for a greater number of high-quality studies, the current quantitative data appeared to support an inverse association between AMC (i.e., total score) and weight status regardless the use of different approaches (process versus product). However, when focusing on AMC, the use of each individual subtest score (locomotor, object control, and stability skills) should be encouraged to acquire a more comprehensive assessment. Locomotor and stability skills seem to be affected by weight status to a greater extent than object control skills during childhood. From a practical perspective, multifaceted activities primarily embedding several running- and jumping-like exercises combined with static and dynamic balance stimuli would help unhealthy-weight children to manage their bodies against gravity, perhaps reducing clumsiness in movements. For instance, it might be assumed that children benefiting from locomotor and stability stimuli would increase their exposure to an active lifestyle within their school years, promoting positive effects on weight status. This may also imply a concurrent increase in their PMC [63], which appeared to be inversely associated with weight status. Giving children an opportunity to experience a range of targeted stimuli might result, in turn, in greater knowledge about movement skills (underlying better motivation and confidence levels), which may concur to prompt them in pursuing physical activities across childhood. Given the current data, the need to target different, specific intervention strategies by gender can be inferred. $M$ children seem to present not only overall superior AMC and PMC compared to F children, but also better individual subtest skills (i.e., locomotor and object control skills), except for stability skills. For instance, F children should be more encouraged to engage in ball games than $\mathrm{M}$ children, with the aim to enhance their mastery of skills involving object control. This could occur both during and outside the school day to increase their hours of deliberate play. Meanwhile, $\mathrm{M}$ children should be continuously exposed to several stimuli with a special emphasis on balancing and rotating movement patterns. In this context, neuromotor training exercises based on balance, agility, motor control, and proprioception could be effective in enhancing M and F children's quality of life (also promoting positive effects on weight status) under both the motor and cognitive domains. Along with training contents based on neuromotor exercises, teaching methods are also crucial to allow $\mathrm{M}$ and F children to explore positive experiences during activity [64]. Specifically, teaching styles with production would lead a child to effectively learn, being free to discover new ways of solving problems related to movement [64], and a reciprocal teaching style would offer to the observer and doer an ongoing chance to record information linked to a motor task with the aim to enhance both AMC and PMC. 
Author Contributions: Conceptualization, A.T., P.L.I. and D.C.; methodology, A.T. and P.L.I.; formal analysis, A.T.; data curation, A.T. and P.L.I.; writing—original draft preparation, A.T., P.L.I., D.M. and D.C.; visualization, A.T. and D.M.; supervision, P.L.I. and D.C. All authors have read and agreed to the published version of the manuscript.

Funding: This research received no external funding.

Institutional Review Board Statement: Not applicable.

Informed Consent Statement: Not applicable.

Data Availability Statement: The datapresented in this study are available on request from the corresponding author.

Conflicts of Interest: The authors declare no conflict of interest.

\section{References}

1. Cioni, G.; Sgandurra, G. Normal Psychomotor Development. Handb. Clin. Neurol. 2013, 111, 3-15.

2. Hilton-Mounger, A. Battelle Developmental Inventory: 2nd Edition. In Encyclopedia of Child Behavior and Development; Goldstein, S., Naglieri, J.A., Eds.; Springer: Boston, MA, USA, 2011; pp. 210-212. ISBN 978-0-387-79061-9.

3. Robinson, L.E.; Stodden, D.F.; Barnett, L.M.; Lopes, V.P.; Logan, S.W.; Rodrigues, L.P.; D’Hondt, E. Motor Competence and its Effect on Positive Developmental Trajectories of Health. Sports Med. 2015, 45, 1273-1284. [CrossRef]

4. Stodden, D.; Langendorfer, S.; Roberton, M.A. The Association Between Motor Skill Competence and Physical Fitness in Young Adults. Res. Q. Exerc. Sport 2009, 80, 223-229. [CrossRef]

5. Morano, M.; Bortoli, L.; Ruiz, M.C.; Campanozzi, A.; Robazza, C. Actual and perceived motor competence: Are children accurate in their perceptions? PLoS ONE 2020, 15, e0233190. [CrossRef]

6. $\quad$ Lopes, V.P.; Malina, R.M.; Lopes, L.; Santos, R.; Stodden, D.F.; Rodrigues, L.P. Testing the Motor Proficiency Barrier Hypothesis for Physical Activity and Weight Status in Youth. J. Sport Health Res. 2021, 13, 103-116.

7. Poulsen, A.A.; Desha, L.; Ziviani, J.; Griffiths, L.; Heaslop, A.; Khan, A.; Leong, G. Fundamental movement skills and self-concept of children who are overweight. Pediatr. Obes. 2011, 6, e464-e471. [CrossRef] [PubMed]

8. Brian, A.; Getchell, N.; True, L.; De Meester, A.; Stodden, D.F. Reconceptualizing and Operationalizing Seefeldt's Proficiency Barrier: Applications and Future Directions. Sports Med. 2020, 50, 1889-1900. [CrossRef]

9. Duncan, M.J.; Jones, V.; O’Brien, W.; Barnett, L.M.; Eyre, E.L.J. Self-Perceived and Actual Motor Competence in Young British Children. Percept. Mot. Ski. 2018, 125, 251-264. [CrossRef]

10. Burns, R.; Kim, Y.; Byun, W.; Brusseau, T.A. Associations of School Day Sedentary Behavior and Physical Activity with Gross Motor Skills: Use of Compositional Data Analysis. J. Phys. Act. Health 2019, 16, 811-817. [CrossRef]

11. Marmeleira, J.; Veiga, G.; Cansado, H.; Raimundo, A. Relationship between motor proficiency and body composition in 6- to 10-year-old children. J. Paediatr. Child Health 2017, 53, 348-353. [CrossRef]

12. Chivers, P. Low Motor Performance Scores among Overweight Children: Poor Coordination or Morphological Constraints? Hum Mov. Sci. 2013, 11, 1127-1137. [CrossRef] [PubMed]

13. Reyes, A.C.; Chaves, R.; Baxter-Jones, A.D.G.; Vasconcelos, O.; Barnett, L.M.; Tani, G.; Hedeker, D.; Maia, J. Modelling the dynamics of children's gross motor coordination. J. Sports Sci. 2019, 37, 2243-2252. [CrossRef]

14. Fintini, D.; Cianfarani, S.; Cofini, M.; Andreoletti, A.; Ubertini, G.M.; Cappa, M.; Manco, M. The Bones of Children with Obesity. Front. Endocrinol. 2020, 11. [CrossRef]

15. Gentier, I.; D’Hondt, E.; Shultz, S.; Deforche, B.; Augustijn, M.; Hoorne, S.; Verlaecke, K.; De Bourdeaudhuij, I.; Lenoir, M. Fine and gross motor skills differ between healthy-weight and obese children. Res. Dev. Disabil. 2013, 34, 4043-4051. [CrossRef] [PubMed]

16. Seefeldt, V. Psychology of motor behavior and sport. In Developmental Motor Patterns: Implications for Elementary School Physical Education; Nadeau, C., Holliwell, W., Roberts, G., Eds.; Human Kinetics: Champaign, IL, USA, 1980.

17. Utesch, T.; Dreiskämper, D.; Naul, R.; Geukes, K. Understanding physical (in-) activity, overweight, and obesity in childhood: Effects of congruence between physical self-concept and motor competence. Sci. Rep. 2018, 8, 5908. [CrossRef]

18. Slotte, S.; Sääkslahti, A.; Kukkonen-Harjula, K.; Rintala, P. Fundamental movement skills and weight status in children: A systematic review. Balt. J. Health Phys. Act. 2017, 9, 115-127. [CrossRef]

19. LeGear, M.; Greyling, L.; Sloan, E.; Bell, R.I.; Williams, B.-L.; Naylor, P.-J.; Temple, A.V. A window of opportunity? Motor skills and perceptions of competence of children in Kindergarten. Int. J. Behav. Nutr. Phys. Act. 2012, 9, 29. [CrossRef]

20. Robinson, L.E. The relationship between perceived physical competence and fundamental motor skills in preschool children. Child Care Health Dev. 2010, 37, 589-596. [CrossRef]

21. Valentini, N.C.; Nobre, G.C.; De Souza, M.S.; Duncan, M. Are BMI, Self-Perceptions, Motor Competence, Engagement, and Fitness Related to Physical Activity in Physical Education Lessons? J. Phys. Act. Health 2020, 17, 493-500. [CrossRef] 
22. Estevan, I.; Menescardi, C.; García-Massó, X.; Barnett, L.M.; Molina-García, J. Profiling children longitudinally: A three-year follow-up study of perceived and actual motor competence and physical fitness. Scand. J. Med. Sci. Sports 2021, 31, 35-46. [CrossRef]

23. Barnett, L.M.; Van Beurden, E.; Morgan, P.J.; Brooks, L.O.; Beard, J.R. Gender Differences in Motor Skill Proficiency from Childhood to Adolescence: A Longitudinal Study. Res. Q. Exerc. Sport 2010, 81, 162-170. [CrossRef] [PubMed]

24. Yang, S.-C.; Lin, S.-J.; Tsai, C.-Y. Effect of Sex, Age, and BMI on the Development of Locomotor Skills and Object Control Skills among Preschool Children. Percept. Mot. Ski. 2015, 121, 873-888. [CrossRef] [PubMed]

25. Moher, D.; Liberati, A.; Tetzlaff, J.; Altman, D.G. Preferred Reporting Items for Systematic Reviews and Meta-Analyses: The PRISMA Statement. BMJ 2009, 339, b2535. [CrossRef] [PubMed]

26. Page, M.J.; Moher, D.; Bossuyt, P.M.; Boutron, I.; Hoffmann, T.C.; Mulrow, C.D.; Shamseer, L.; Tetzlaff, J.M.; Akl, E.A.; Brennan, E.S.; et al. PRISMA 2020 explanation and elaboration: Updated guidance and exemplars for reporting systematic reviews. BMJ 2021, 372, n160. [CrossRef] [PubMed]

27. Von Elm, E.; Altman, D.G.; Egger, M.; Pocock, S.J.; Gøtzsche, P.C.; Vandenbroucke, J.P. The Strengthening the Reporting of Observational Studies in Epidemiology (STROBE) Statement: Guidelines for Reporting Observational Studies. J. Clin. Epidmiol. 2008, 61, 344-349. [CrossRef]

28. Moher, D.; Schulz, K.F.; Altman, D.G. The CONSORT Statement: Revised Recommendations for Improving the Quality of Reports of Parallel-Group Randomised Trials. Lancet 2001, 357, 1191-1194. [CrossRef]

29. Lubans, D.R.; Morgan, P.; Cliff, D.; Barnett, L.M.; Okely, A. Fundamental Movement Skills in Children and Adolescents. Sports Med. 2010, 40, 1019-1035. [CrossRef] [PubMed]

30. Bryant, E.S.; Duncan, M.; Birch, S.L. Fundamental movement skills and weight status in British primary school children. Eur. J. Sport Sci. 2014, 14, 730-736. [CrossRef] [PubMed]

31. Bryant, E.S.; James, R.S.; Birch, S.L.; Duncan, M.; Duncan, M. Prediction of habitual physical activity level and weight status from fundamental movement skill level. J. Sports Sci. 2014, 32, 1775-1782. [CrossRef]

32. Cliff, D.P.; Okely, A.; Magarey, A. Movement skill mastery in a clinical sample of overweight and obese children. Pediatr. Obes. 2011, 6, 473-475. [CrossRef]

33. Comeau, M.E.; Bouchard, D.R.; Levesque, C.; Jonhson, M.J.; Rioux, B.V.; Mayo, A.; Sénéchal, M. Association between Functional Movements Skills and Health Indicators in Children Aged between 9 and 12 Years Old. Int. J. Environ. Res. Public Health 2017, 14, 1010. [CrossRef] [PubMed]

34. De Meester, A.; Stodden, D.; Brian, A.; True, L.; Cardon, G.; Tallir, I.; Haerens, L. Associations among Elementary School Children's Actual Motor Competence, Perceived Motor Competence, Physical Activity and BMI: A Cross-Sectional Study. PLoS ONE 2016, 11, e0164600. [CrossRef]

35. Duncan, M.J.; Bryant, E.; Stodden, D. Low fundamental movement skill proficiency is associated with high BMI and body fatness in girls but not boys aged 6-11 years old. J. Sports Sci. 2017, 35, 2135-2141. [CrossRef]

36. Duncan, M.J.; Stanley, M. Functional Movement Is Negatively Associated with Weight Status and Positively Associated with Physical Activity in British Primary School Children. J. Obes. 2012, 2012, 697563. [CrossRef]

37. Hardy, L.L.; Reinten-Reynolds, T.; Espinel, P.; Zask, A.; Okely, A.D. Prevalence and Correlates of Low Fundamental Movement Skill Competency in Children. Pediatrics 2012, 130, e390-e398. [CrossRef] [PubMed]

38. Kelly, L.; O'Connor, S.; Harrison, A.J.; Chéilleachair, N.N. Does fundamental movement skill proficiency vary by sex, class group or weight status? Evidence from an Irish primary school setting. J. Sports Sci. 2019, 37, 1055-1063. [CrossRef] [PubMed]

39. Spessato, B.C.; Gabbard, C.; Robinson, L.E.; Valentini, N. Body mass index, perceived and actual physical competence: The relationship among young children. Child Care Health Dev. 2013, 39, 845-850. [CrossRef] [PubMed]

40. Augustijn, M.J.C.M.; Deconinck, F.J.A.; D’Hondt, E.; Van Acker, L.; De Guchtenaere, A.; Lenoir, M.; Caeyenberghs, K. Reduced motor competence in children with obesity is associated with structural differences in the cerebellar peduncles. Brain Imaging Behav. 2018, 12, 1000-1010. [CrossRef] [PubMed]

41. Cheng, J.; East, P.; Blanco, E.; Sim, E.K.; Castillo, M.; Lozoff, B.; Gahagan, S. Obesity leads to declines in motor skills across childhood. Child Care Health Dev. 2016, 42, 343-350. [CrossRef]

42. Chowdhury, S.D.; Wrotniak, B.H.; Ghosh, T. Association Between Body Mass Index and Motor Competence in Santal Children of Purulia District, India. J. Mot. Behav. 2017, 49, 349-354. [CrossRef]

43. Coppens, E.; Bardid, F.; Deconinck, F.J.A.; Haerens, L.; Stodden, D.; D’Hondt, E.; Lenoir, M. Developmental Change in Motor Competence: A Latent Growth Curve Analysis. Front. Physiol. 2019, 10, 1273. [CrossRef] [PubMed]

44. Moura-Dos-Santos, M.A.; De Almeida, M.B.; Manhães-De-Castro, R.; Katzmarzyk, P.T.; Maia, J.A.R.; Leandro, C.G. Birthweight, Body Composition, and Motor Performance in 7- to 10-Year-Old Children. Dev. Med. Child. Neurol. 2015, 57, 470-475. [CrossRef] [PubMed]

45. D’Hondt, E.; Deforche, B.; Vaeyens, R.; Vandorpe, B.; Vandendriessche, J.; Pion, J.; Philippaerts, R.; De Bourdeaudhuij, I.; Lenoir, M. Gross motor coordination in relation to weight status and age in 5- to 12-year-old boys and girls: A cross-sectional study. Pediatr. Obes. 2011, 6, e556-e564. [CrossRef]

46. D'Hondt, E.; Deforche, B.; Gentier, I.; De Bourdeaudhuij, I.; Vaeyens, R.; Philippaerts, R.; Lenoir, M. A longitudinal analysis of gross motor coordination in overweight and obese children versus normal-weight peers. Int. J. Obes. 2013, 37, 61-67. [CrossRef] [PubMed] 
47. D'Hondt, E.; Gentier, I.; Deforche, B.; Tanghe, A.; De Bourdeaudhuij, I.; Lenoir, M. Weight Loss and Improved Gross Motor Coordination in Children as a Result of Multidisciplinary Residential Obesity Treatment. Obesity 2011, 19, 1999-2005. [CrossRef]

48. Laukkanen, A.; Bardid, F.; Lenoir, M.; Lopes, V.P.; Vasankari, T.; Husu, P.; Sääkslahti, A. Comparison of motor competence in children aged 6-9 years across northern, central, and southern European regions. Scand. J. Med. Sci. Sports 2020, 30, 349-360. [CrossRef] [PubMed]

49. Lima, R.A.; Bugge, A.; Ersbøll, A.K.; Stodden, D.F.; Andersen, L.B. The longitudinal relationship between motor competence and measures of fatness and fitness from childhood into adolescence. J. Pediatr. 2019, 95, 482-488. [CrossRef]

50. Lopes, V.P.; Malina, R.M.; Maia, J.A.R.; Rodrigues, L.P. Body mass index and motor coordination: Non-linear relationships in children 6-10 years. Child Care Health Dev. 2018, 44, 443-451. [CrossRef]

51. Lopes, L.; Santos, R.; Moreira, C.; Pereira, B.; Lopes, V.P. Sensitivity and specificity of different measures of adiposity to distinguish between low/high motor coordination. J. Pediatr. 2015, 91, 44-51. [CrossRef]

52. Morrison, K.M.; Cairney, J.; Eisenmann, J.; Pfeiffer, K.; Gould, D. Associations of Body Mass Index, Motor Performance, and Perceived Athletic Competence with Physical Activity in Normal Weight and Overweight Children. J. Obes. 2018, 2018, 3598321. [CrossRef]

53. Rodrigues, L.P.; Stodden, D.; Lopes, V.P. Developmental pathways of change in fitness and motor competence are related to overweight and obesity status at the end of primary school. J. Sci. Med. Sport 2016, 19, 87-92. [CrossRef]

54. Cattuzzo, M.T.; Henrique, R.D.S.; Ré, A.; de Oliveira, I.S.; Melo, B.M.; Moura, M.D.S.; de Araújo, R.C.; Stodden, D. Motor competence and health related physical fitness in youth: A systematic review. J. Sci. Med. Sport 2016, 19, 123-129. [CrossRef] [PubMed]

55. Hulteen, R.M.; True, L.; Pfeiffer, K.A. Differences in associations of product- and process-oriented motor competence assessments with physical activity in children. J. Sports Sci. 2020, 38, 375-382. [CrossRef] [PubMed]

56. Logan, S.W.; Barnett, L.M.; Goodway, J.D.; Stodden, D. Comparison of performance on process- and product-oriented assessments of fundamental motor skills across childhood. J. Sports Sci. 2017, 35, 634-641. [CrossRef]

57. Stodden, D.; Goodway, J.D.; Langendorfer, S.J.; Roberton, M.A.; Rudisill, M.E.; Garcia, C.; Garcia, L.E. A Developmental Perspective on the Role of Motor Skill Competence in Physical Activity: An Emergent Relationship. Quest 2008, 60, 290-306. [CrossRef]

58. Khodaverdi, Z.; Bahram, A.; Stodden, D.; Kazemnejad, A. The relationship between actual motor competence and physical activity in children: Mediating roles of perceived motor competence and health-related physical fitness. J. Sports Sci. 2016, 34, 1523-1529. [CrossRef]

59. Barnett, L.M.; Morgan, P.J.; Van Beurden, E.; Beard, J.R.; Barnett, L.M.; Morgan, P.J.; Van Beurden, E.; Beard, J.R. Perceived sports competence mediates the relationship between childhood motor skill proficiency and adolescent physical activity and fitness: A longitudinal assessment. Int. J. Behav. Nutr. Phys. Act. 2008, 5, 40. [CrossRef]

60. Hall, C.; Eyre, E.; Oxford, S.; Duncan, M. Does Perception of Motor Competence Mediate Associations between Motor Competence and Physical Activity in Early Years Children? Sports 2019, 7, 77. [CrossRef]

61. Madrona, P.G.; Carrillo-López, P.J.; Cantó, E.G.; Guillamón, A.R. Incidencia del sexo, el índice de masa corporal y el número de hermanos en la competencia motriz autopercibida. Rev. Cuba Investig. Bioméd. 2020, 39, e370.

62. Bardid, F.; Huyben, F.; Lenoir, M.; Seghers, J.; De Martelaer, K.; Goodway, J.D.; Deconinck, F.J.A. Assessing fundamental motor skills in Belgian children aged 3-8 years highlights differences to US reference sample. Acta Paediatr. 2016, 105, e281-e290. [CrossRef]

63. Madrona, P.G.; Lopez, P.J.C.; Guillamón, A.R.; Cantó, E.G.; Ghorbani, S. Percepción de competencia física en escolares de España e Irán: Un estudio cultural. Educación Física y Deporte 2019, 38. [CrossRef]

64. Mosston, M.; Ashworth, S. Teaching Physical Education; Benjamin-Cummings Pub Co: San Francisco, CA, USA, 2002. 